\title{
Performance of Linear Field Reconstruction Techniques With Noise and Uncertain Sensor Locations
}

\author{
Alessandro Nordio, Member, IEEE, Carla-Fabiana Chiasserini, Member, IEEE, and
} Emanuele Viterbo, Senior Member, IEEE

\begin{abstract}
We consider a wireless sensor network, sampling a bandlimited field, described by a limited number of harmonics. Sensor nodes are irregularly deployed over the area of interest or subject to random displacement; in addition sensors measurements are affected by noise. Our goal is to obtain a high quality reconstruction of the field, with the mean square error (MSE) of the estimate as performance metric. In particular, we analytically derive the performance of several reconstruction/estimation techniques based on linear filtering. For each technique, we obtain the MSE, as well as its asymptotic expression in the case where the number of field-harmonics and the number of sensors grow to infinity, while their ratio is kept constant. Through numerical simulations, we show the validity of the asymptotic analysis, even for a small number of sensors. We provide some novel guidelines for the design of sensor networks when many parameters, such as field bandwidth, number of sensors, reconstruction quality, and sensor displacement characteristics, to be traded off.
\end{abstract}

Index Terms-Irregular sampling, linear filtering, sensor networks.

\section{INTRODUCTION}

W IRELESS sensor networks are often used for applications like environmental and traffic control, habitat monitoring, or weather forecasts [1], which require to sample a physical phenomenon over an area of interest (the sensor field). In this paper, we consider a set of sensors communicating with a sink node, through either single- or multihop communications. Each sensor locally samples the physical field, while the sink collecting all samples is in charge of reconstructing the signal of interest.

We assume that initially sensors are either located at predefined positions, or, if randomly deployed over the network area, their location can be estimated at the sink node [2]. We do not deal with spatiotemporal correlation, but consider a fixed time instant and focus on the spatial sampling and reconstruction of the sensor field. We note that, in general, sensors provide an irregular sampling of the observed phenomenon. This may be

Manuscript received October 4, 2006; revised March 31, 2008. This work was supported by the Italian Minister through the FAR project MEADOW. The associate editor coordinating the review of this manuscript and approving it for publication was Dr. Zoran Cvetkovic.

A. Nordio and C.-F. Chiasserini are with the Department of Electronic Engineering, Politecnico di Torino, 10129 Torino, Italy (e-mail: alessandro.nordio@polito.it; carla.chiasserini@ polito.it).

E. Viterbo is with the Dipartimento di Elettronica, Informatica e Sistemistica (DEIS), Università della Calabria, 87036 Rende (CS), Italy (e-mail: viterbo@deis.unical.it).

Digital Object Identifier 10.1109/TSP.2008.924865 due to various reasons: random deployment of the nodes, environment characteristics that bias the network deployment, sensors entering a sleep mode, inaccuracy in sensor positioning, or nodes movement [3]. In all these cases, the sink has to reconstruct the field from a collection of samples that are irregularly spaced, different from the classical equally (or regularly) spaced sampling.

The problem of signal reconstruction from irregular samples has been widely addressed in signal processing, where several efficient and fast algorithms have been proposed to numerically reconstruct or approximate a signal [4]-[6]. The problem we address in this paper, however, is different; the questions we pose are as follows:

(i) How do noisy measures and inaccurate knowledge of the sensor positions affect the reconstruction quality?

(ii) How can we trade off system parameters like measurement noise, field bandwidth, signal reconstruction quality, and number of sensors?

We answer these questions by using a probabilistic approach. Specifically, we analyze two different models of the monitoring system that account for the quality of the measurements performed by the sensors and differ in the accuracy with which the sensor positions are known at the sink node. The model denoted as Model A refers to the case where sensors are fixed, the sink has perfect knowledge of the sensor positions, but the sensor measurements are affected by error. In the second model, named Model B, besides noisy measurements, we consider that the sensor positions vary around an average value, and only the average location of the nodes is known at the sink. Examples where this model applies are observation systems using surface buoys [7], underwater robots located at different depths [8], dropsondes, or low-cost unmanned platforms [9]. Clearly, Model B reduces to Model A when there is no uncertainty about the sensor positions.

For each of these models, we use as field reconstruction techniques some linear filters that are commonly employed in signal detection and estimation, and we evaluate the mean square error (MSE) of the resulting estimate. Interestingly, so far the link between linear filters theory and reconstruction of irregularly sampled fields has not been deeply investigated in the literature.

We find that a key parameter for the network performance is the ratio $\beta$ of the number of field-harmonics to the number of sampling sensors. In particular, there exists a value of this ratio, beyond which the performance of all considered reconstruction strategies degrade significantly, even for low values of 
noise level and limited uncertainty on the sensor positions. To obtain an acceptable reconstruction quality when $\beta$ is large (i.e., the number of available sensors is limited compared to the field bandwidth), reconstruction techniques that exploit some knowledge of the measurement noise and of the jitter in the sensors position must be employed.

We also carry out an asymptotic analysis of the system as the number of field-harmonics and the number of sensors grow to infinity, while their ratio $\beta$ is kept constant, and we show that this is an effective tool to study the system performance [10], even when the number of sensors is small. Finally, we find a lower bound to the MSE that can be achieved by any of the considered techniques, both under Model A and Model B.

In summary, the novelty of this paper is based on the probabilistic approach to the performance analysis of linear reconstruction algorithms. This analysis can be carried on analytically thanks to the linearity of the considered reconstruction techniques.

The remainder of the paper is organized as follows. In Section II we present our assumptions and the system models under study. Section III discusses some related work. Section IV introduces the performance metrics and provides some mathematical tools necessary for our study. Model A and B are analyzed in Sections V and VI, respectively. Finally, Sections VII and VIII summarize our main results and draw some conclusions.

\section{ASSUMPTIONS AND SYSTEM MODELS}

Let us consider a one-dimensional field. A physical field is approximately bandlimited and, when observed over a finite interval, it admits an infinite Fourier series expansion. However, one can think of the largest index $M$ of the non-negligible Fourier coefficients of the expansion as the approximate one-sided bandwidth of the field. We therefore represent the one-dimensional field, $s(x)$, by using $2 M+1$ harmonics as

$$
s(x)=\frac{1}{\sqrt{2 M+1}} \sum_{k=-M}^{M} a_{k} \mathrm{e}^{\mathrm{j} 2 \pi k x} .
$$

The field is observed within one period interval $[0,1)$ and sampled by $r$ sensors placed at positions ${ }^{1} \mathbf{x}=\left[x_{1}, \ldots, x_{r}\right]^{\mathrm{T}}$, $x_{q} \in[0,1), q=1, \ldots, r$, which are in general not equally spaced. The signal samples are denoted by the column vector $\mathbf{s}=\left[s\left(x_{1}\right), \ldots, s\left(x_{r}\right)\right]^{\mathrm{T}}$. The field discrete spectrum is given by the $2 M+1$ complex vector $\mathbf{a}=\left[a_{-M}, \ldots, a_{0}, \ldots, a_{M}\right]^{T}$. The complex numbers $a_{k}$ represent amplitudes and phases of the harmonics in $s(x)$.

We assume that the entries of $\mathbf{x}$ are i.i.d. uniformly distributed random variables in $[0,1)$. The extension to a multidimensional field can be easily obtained, as discussed later in this section.

We define $\beta$ as the ratio of the number of harmonics which describe the field to the number of sensors, i.e., $\beta=(2 M+$ $1) / r$. This is an important parameter in our analysis. Note that the number of sensors $r$ also corresponds to the sampling rate;

\footnotetext{
${ }^{1}$ Column vectors and matrices are denoted by bold lowercase and bold upper case letters, respectively. $(\mathbf{X})_{k q}$ is the $(k, q)$ entry of the matrix $\mathbf{X}, \mathbf{I}_{n}$ is the $n \times$ $n$ identity matrix, and $\mathbf{I}$ is the generic identity matrix. The conjugate transpose operator is denoted by $(\cdot)^{\dagger}$.
}

thus, the number $\beta$ is the ratio of twice the field bandwidth to the sampling rate (frequency). In particular, in regular sampling theory, exact reconstruction is achieved for $0 \leq \beta<1$ and, if a Nyquist regular sampling interval were used, we would have: $\beta=1$.

In the next sections, we derive asymptotic results in the case where the number $2 M+1$ of field-harmonics and the number $r$ of sensors both grow to infinity, while their ratio $\beta$ is kept constant.

Notice that this implicitly requires making some assumptions about the existence of the asymptotic limit of the signal in (1). A study of sufficient conditions which ensure asymptotic model consistency though important and interesting is outside the scope of the present work. In this context, the readers are referred to the literature on nonparametric regression with random (Monte Carlo) sampling [11], [12], which is closely related to the present problem.

We consider $M$ to be known, and the random vector a to have zero mean and covariance matrix $\mathbb{E}\left[\mathbf{a a}^{\dagger}\right]=\sigma_{a}^{2} \mathbf{I}_{2 M+1}$, where $\sigma_{a}^{2}$ corresponds to the field average power spectral density. The value $\mathbf{s}$ of the field at positions $\mathbf{x}$ depends on the spectrum $\mathbf{a}$ through the expression

$$
\mathbf{s}=\mathbf{G}_{\mathbf{x}}^{\dagger} \mathbf{a}
$$

where $\mathbf{G}_{\mathbf{X}}$ is the $(2 M+1) \times r$ generalized Fourier matrix defined as

$$
\left(\mathbf{G}_{\mathbf{x}}\right)_{k q}=\frac{1}{\sqrt{2 M+1}} \mathrm{e}^{-\mathrm{j} 2 \pi k x_{q}} \quad k=-M, \ldots, M q=1, \ldots, r .
$$

The dependence of the matrix $\mathbf{G}_{\mathbf{x}}$ on the position vector $\mathbf{x}$ is clearly indicated by its subscript. When the samples are equally spaced in the interval $[0,1)$, the matrix $\sqrt{\beta} \mathbf{G}_{\mathbf{X}}$ is a unitary matrix (i.e., $\beta \mathbf{G}_{\mathbf{x}} \mathbf{G}_{\mathbf{x}}^{\dagger}=\mathbf{I}_{2 M+1}$ ). The above system model refers to a unidimensional field where sensor positions are determined by a scalar variable. However, the extension to the multidimensional case can still be easily obtained since the relation between field spectrum and samples in a band-limited multidimensional field can be expressed in a matrix form similar to (2), where only the structure of the matrix $\mathbf{G}_{\mathbf{x}}$ differs.

Finally, we assume that sensor field measures are sent to a processing unit, the so-called sink node, whose task is to provide an estimate of the sensed field. Since we focus on the reconstruction of the physical field, we consider that sensor transmissions always reach successfully the sink node. By relying on the assumptions discussed above, we study the following two systems.

Model A: Fixed sensors, perfect knowledge of the sensor positions, noisy measures: In this model, sensors have a fixed position, given by the vector $\mathbf{x}$ and known at the sink node, but each sensor provides a measure of the field affected by additive noise with zero mean and variance $\sigma_{n}^{2}$ [13]. The additive noise approximates the errors affecting the measurement procedure [14]. The measures can, therefore, be written as

$$
\mathbf{p}=\mathbf{s}+\mathbf{n}=\mathbf{G}_{\mathbf{x}}^{\dagger} \mathbf{a}+\mathbf{n}
$$

where $\mathbf{s}$ is the sampled field and the zero mean noise vector is denoted by $\mathbf{n}$, with covariance matrix $\mathbb{E}\left[\mathbf{n n}^{\dagger}\right]=\sigma_{n}^{2} \mathbf{I}_{r}$. 
Model B: Sensors with jittered positions and noisy measures: In this case, each sensor displaced around an average position $\hat{x}_{q}(q=1, \ldots, r)$, i.e., the sensor positions are given by: $\mathbf{x}=\hat{\mathbf{x}}+\boldsymbol{\delta}$, where $\mathbb{E}[\mathbf{x}]=\hat{\mathbf{x}}$ and $\boldsymbol{\delta}$ is the displacement of the sensors with respect to their average location $\hat{\mathbf{x}}$. Notice that this problem is related to the problem of jittered sampling which has been investigated in many works dealing with both regular and irregular sampling [15].

The displacements $\delta_{q}, q=1, \ldots, r$, are modeled as independent zero mean Gaussian random variables with variance $\sigma_{\delta}^{2}$ and $\mathbb{E}\left[\delta \boldsymbol{\delta}^{\dagger}\right]=\sigma_{\delta}^{2} \mathbf{I}_{r}$. For convenience and neglecting the edge effects, we consider $\bmod \left(x_{q}, 1\right)$ so that $x_{q}$ falls in the observation interval $[0,1)$. The vector $\mathbf{p}$ of measures is still given by (4). Also, noise, displacement, and field spectrum are assumed to be uncorrelated, hence, $\mathbb{E}\left[\mathbf{n} \boldsymbol{\delta}^{\dagger}\right]=\mathbb{E}\left[\mathbf{n a}^{\dagger}\right]=\mathbb{E}\left[\mathbf{a} \delta^{\dagger}\right]=\mathbf{0}$, and the sink has perfect knowledge of $\hat{\mathbf{x}}$.

\section{RELATED WORK}

The problem of sampling a physical field through a wireless sensor network has been widely addressed in the literature. In particular, several papers deal with energy efficiency issues, aiming at prolonging the network lifetime as much as possible. The work in [16] considers that sensors can enter a low-power operational state (i.e., a sleep mode) and presents an algorithm to determine which sensor subsets should be selected to acquire data from an area of interest and which nodes should remain inactive to save energy. Note that in our paper we consider an irregular topology, which may be caused by nodes moving into a sleep state; however, we do not directly address energy efficiency or scheduling of the node sleep/activity periods.

In [17], the authors consider a uni-dimensional field, uniformly oversampled at a multiple of the Nyquist frequency by low-precision sensors. The impact on the field reconstruction accuracy of quantization errors and node density is evaluated. The effect of random error sources affecting the ADC, besides quantization, is investigated in [14]. In our work we consider an additive noise that models errors due to the measurement procedure as well as errors due to the ADC, but we do not specifically focus on the latter issue.

The impact of medium access control protocols on the field reconstruction field is investigated in [18], [19]. In particular, the paper in [19] considers a dense sensor network and analyzes the impact of deterministic and random data collection strategies on the quality of field reconstruction. As a performance metric, the maximum of the reconstruction square error over the sensed field is adopted, as opposed to our work where the MSE is considered. Also, in [19] the field is a Gaussian random process, the exact sensor locations are known and the central controller always receives a sufficiently large number of samples.

The field reconstruction at the sink node, with spatial and temporal correlation among sensor measures, is studied in [13], [20]-[22]. In [22], the observed field is a discrete vector of target positions and they assume correlated sensor observations. By modeling the sensor network as a channel encoder and exploiting some concepts from coding theory, the network capacity, defined as the maximum ratio of target positions to number of sensors, is studied as a function of noise, sensing function and sensor connections. The problem of reconstructing a band-limited signal from an irregular set of samples at unknown locations is addressed in [23]. There, the signal is oversampled by irregularly spaced sensors; sensor positions are unknown but always equal to an integer multiple of the sampling interval. Differently from [23], we assume that the sink can either acquire or estimate the sensor locations and that sensors are randomly deployed over a finite interval.

Related to our work is the literature on spectral analysis (see, e.g., [24] and references therein). In this field, an excellent guide to irregular sampling is [6], which covers a large number of reconstruction techniques, considering a wide range of applications. Reconstruction techniques for irregularly or randomly sampled signals include, among others, linear [4] and nonlinear [25] methods, iterative algorithms [26], and interpolation. In particular, Feichtinger and Gröchenig in [26] provide an error analysis of an iterative reconstruction algorithm taking into account round-off errors, jitters, truncation errors, and aliasing. Other theoretical works on irregular sampling can be found in [4], [5], [25]-[29], and references therein. The main differences between our work and the results that are available in the spectral analysis literature are the following:

(i) the analysis of the asymptotic MSE, where $M$ and $r$ go to infinity keeping their ratio $\beta$ fixed;

(ii) the application of results from the theory of random matrices to the field reconstruction problem;

(iii) the focus on a Bayesian model for the (random) sensor locations, as opposed to the frequentist model of deterministic unknown locations that one encounters more commonly in the spectral analysis literature.

Finally, in our previous work [30] some conditions on the irregular topology of the sensor network are identified, which allow for a successful signal reconstruction, both under deterministic and random node deployment. In particular, in [30] the spectrum estimate $\hat{a}$, computed by the sink, is obtained by applying to $\mathbf{s}$ the Moore-Penrose pseudoinverse of the matrix $\mathbf{G}_{\mathbf{x}}$, i.e., $\hat{\mathbf{a}}=\left(\mathbf{G}_{\mathbf{x}} \mathbf{G}_{\mathbf{x}}^{\dagger}\right)^{-1} \mathbf{G}_{\mathbf{x}} \mathbf{s}$. The system model adopted in [30] is ideal in the sense that the reconstruction algorithm has perfect knowledge of the vector $\mathbf{x}$ and neglects noisy measures: the failure in reconstruction (i.e., $\hat{\mathbf{a}} \neq \mathbf{a}$ ) is only due to the bad conditioning of the matrix $\mathbf{G}_{\mathbf{x}} \mathbf{G}_{\mathbf{x}}^{\dagger}$ in relation to the finite machine precision. In this paper, instead, we apply linear filters to the field reconstruction and consider the following causes of quality degradation: (i) noisy measures and (ii) uncertainty at the sink on the sensors position.

\section{PRELIMINARIES}

Here we describe the techniques we use for field reconstruction, and define the performance metrics employed for assessing the effectiveness of these techniques on the quality of the reconstructed field. Finally, we provide some mathematical tools necessary for the analysis of the models under study.

\section{A. Reconstruction Techniques}

Several reconstruction techniques have been proposed in the literature, which amount to the solution of a linear system (see [4], [5], and references therein). A widely used technique consists in processing the measures $\mathbf{p}$ by means of a linear filter $\mathbf{B}$, which is an $r \times(2 M+1)$ matrix and is a function of the system 
parameters known at the sink. In this case, the estimate of the field spectrum is given by

$$
\hat{\mathbf{a}}=\mathbf{B}^{\dagger} \mathbf{p} \text {. }
$$

The system model in (4) is similar to the one employed in multiuser communications [31] or multiple antennas communications [32], [33]. In those cases $\mathbf{p}$ is the received signal, the matrix $\mathbf{G}_{\mathbf{x}}$ plays the role of spreading matrix or channel matrix, a is the transmitted signal and $\mathbf{n}$ is the channel noise. By relying on the results obtained in those fields, for each system model we propose and compare some reconstruction techniques characterized by different matrices $\mathbf{B}$ : the matched filter $(M F)$, the zero forcing $(Z F)$ filter, and some linear filters minimizing the MSE (LMMSE) [31]. In the field of multiuser detection, the MF simply correlates the received signal with the desired user's time reversed spreading waveform, thus, it does not take into account any other users in the system or channel dynamics. The ZF filter counteracts multiuser interference but it ignores the presence of channel noise. The LMMSE solution minimizes the squared error between the received and transmitted signals, thus, accounting also for the channel noise; it becomes the zero-forcing solution when no noise is present. Note that the advantage of the MF with respect to the ZF and LMMSE filters is that no matrix inversion is needed; while between the ZF and the LMMSE filter, clearly the best performance in terms of minimum square error is given by the LMMSE, however, the advantage of the $\mathrm{ZF}$ filter is that it does not require any knowledge of the noise component (see [31] for further details).

\section{B. Performance Metrics}

Given the spectrum estimate (5), the field can be reconstructed as $\hat{s}(x)=(2 M+1)^{-1 / 2} \sum_{k=-M}^{M} \hat{a}_{k} \exp (\mathrm{j} 2 \pi k x)$. As a measure of the reconstruction quality, we consider the MSE of the estimate of $s(x)$, which is given by $\mathrm{MSE}=\mathbb{E}\left[\int_{0}^{1}|s(x)-\hat{s}(x)|^{2} \mathrm{~d} x\right]$. We observe that computing MSE as above is equivalent to computing $\mathbb{E}\left[\|\mathbf{a}-\hat{\mathbf{a}}\|^{2}\right]$. Indeed, by substituting (1) and the expression of $\hat{s}(x)$, we have

$$
\int_{0}^{1}|s(x)-\hat{s}(x)|^{2} \mathrm{~d} x=\frac{1}{2 M+1}\|\mathbf{a}-\hat{\mathbf{a}}\|^{2} .
$$

Thus, in the following, for a given vector of sensor positions $\mathbf{x}$, we consider the MSE defined as

$$
\mathrm{MSE}_{\mathbf{x}}=\frac{\mathbb{E}\left[\|\hat{\mathbf{a}}-\mathbf{a}\|^{2}\right]}{2 M+1}=\frac{\sigma_{a}^{2}}{2 M+1} \operatorname{Tr}\left\{\boldsymbol{\Psi}_{\mathbf{x}}\right\}
$$

where

$$
\Psi_{\mathbf{x}} \triangleq \frac{1}{\sigma_{a}^{2}} \mathbb{E}\left[(\hat{\mathbf{a}}-\mathbf{a})(\hat{\mathbf{a}}-\mathbf{a})^{\dagger}\right]
$$

is a $(2 M+1) \times(2 M+1)$ matrix, the operator $\mathbb{E}[\cdot]$ averages with respect to all random variables of the model, and $\operatorname{Tr}\{\cdot\}$ is the trace operator. Also, in (6) we exploited the fact that, for any vector $\mathbf{v}$, we have: $\mathbb{E}\left[\|\mathbf{v}\|^{2}\right]=\mathbb{E}\left[\operatorname{Tr}\left\{\mathbf{v} \mathbf{v}^{\dagger}\right\}\right]=\operatorname{Tr}\left\{\mathbb{E}\left[\mathbf{v} \mathbf{v}^{\dagger}\right]\right\}$.
Next, we consider the vector $\mathbf{x}$ to be random. In this case a more appropriate performance metric is the average MSE, normalized to $\sigma_{a}^{2}$, i.e., $\mathrm{MSE}_{\mathrm{av}}=\underset{\mathbf{x}}{\mathbb{E}}\left[\mathrm{MSE}_{\mathbf{x}}\right] / \sigma_{a}^{2}$ where $\mathrm{MSE}_{\mathbf{x}}$ is as in (6) and $\underset{\mathbf{x}}{\mathbb{E}}[\cdot]$ averages over the realizations of $\mathbf{x}$.

When the parameters $M$ and $r$ grow to infinity while the ratio $\beta=(2 M+1) / r$ is kept constant, we define the asymptotic average MSE as

$$
\mathrm{MSE}_{\infty}=\lim _{\substack{M, r \rightarrow+\infty \\ \beta}} \mathrm{MSE}_{\mathrm{av}}
$$

The results presented later in the paper show that $\mathrm{MSE}_{\infty}$ gives a very good approximation of $\mathrm{MSE}_{a v}$, already for small values of $M$. This is a common feature of asymptotic analysis based on random matrices [32].

\section{Some Mathematical Tools}

1) The Functional $\phi$ : Let us first consider an $n \times n$ Hermitian random matrix $\mathbf{X}$ and the functional $\phi(\mathbf{X}) \triangleq$ $\lim _{n \rightarrow+\infty}(1 / n) \mathbb{E}[\operatorname{Tr}\{\mathbf{X}\}]$. Using (7) and (8), the asymptotic MSE can be written as

$$
\mathrm{MSE}_{\infty}=\phi\left(\boldsymbol{\Psi}_{\mathbf{x}}\right)
$$

In our analysis we use the following results on the functional $\phi(\cdot)$. First, we notice that $\phi(\mathbf{I})=1$. Second, we can prove (see Appendix I) that, if $g(x)$ is an analytic function defined in $x>0$, then $^{2}$

$$
\phi(g(\mathbf{X}))=\mathbb{E}[g(\xi)]
$$

where $\xi$ is a random variable with the asymptotic eigenvalue distribution of $\mathbf{X}$.

2) A Simple Expression for $\mathbf{G}_{\hat{\mathbf{x}}+\delta}$ : As will be clear in Section VI, in the analysis of Model B many parameters are functions of the matrix $\mathbf{G}_{\mathbf{x}}$, where $\mathbf{x}=\hat{\mathbf{x}}+\boldsymbol{\delta}$. It is, thus, useful to derive an expression of $\mathbf{G}_{\mathbf{x}}$ as a function of $\mathbf{G}_{\hat{\mathbf{x}}}$, in order to separate the random part $\boldsymbol{\delta}$ of $\mathbf{x}$ from the constant part $\hat{\mathbf{x}}$. A useful expression of $\mathbf{G}_{\hat{\mathbf{x}}}$ in terms of $\mathbf{G}_{\mathbf{x}}$ is given here.

Lemma IV.1: For any vector $\mathbf{x}$ of size $r$, let the $(k, q)$ entry of the matrix $\mathbf{G}_{\mathbf{x}}$ be $\left(\mathbf{G}_{\mathbf{x}}\right)_{k q}=(2 M+1)^{-1 / 2} \exp \left(-\mathrm{j} 2 \pi k x_{q}\right)$ for $k=-M, \ldots, M$, and $q=1, \ldots, r$. Let the size $r$ column vectors $\mathbf{x}, \hat{\mathbf{x}}$, and $\boldsymbol{\delta}$ be such that $\mathbf{x}=\hat{\mathbf{x}}+\boldsymbol{\delta}$, then

$$
\mathbf{G}_{\mathbf{x}}=\sum_{n=0}^{\infty} \frac{1}{n !} \mathbf{W}^{n} \mathbf{G}_{\hat{\mathbf{x}}} \Delta^{n}
$$

where $\boldsymbol{\Delta}=\operatorname{diag}(\boldsymbol{\delta})$ is an $r \times r$ diagonal matrix, and $\mathbf{W}$ is a $(2 M+1) \times(2 M+1)$ diagonal matrix with $(\mathbf{W})_{k k}=-\mathrm{j} 2 \pi k$.

The proof is given in Appendix II.

\section{ANALYSIS OF MODEL A}

Here we consider the case where sensor positions are fixed and known at the sink but the field estimates are degraded by noisy measures. We analyze three different linear filters: the matched filter, the zero forcing filter and the minimum MSE

${ }^{2}$ Note the small abuse of notation when using $g(\cdot)$ for both scalar and matrix argument. 
filter [31]. In all cases, for any fixed $\mathbf{x}$, the filter matrix $\mathbf{B}$ is deterministic. Using (4), (5), and (7), we have

$$
\begin{aligned}
\boldsymbol{\Psi}_{\mathbf{x}} & =\frac{1}{\sigma_{a}^{2}} \mathbb{E}_{\mathbf{a}, \mathbf{n}}\left[\|\hat{\mathbf{a}}-\mathbf{a}\|^{2}\right] \\
& =\frac{1}{\sigma_{a}^{2}} \mathbb{E}_{\mathbf{a}, \mathbf{n}}\left[\left\|\mathbf{B}^{\dagger}\left(\mathbf{G}_{\mathbf{x}}^{\dagger} \mathbf{a}+\mathbf{n}\right)-\mathbf{a}\right\|^{2}\right] \\
& =\left(\mathbf{B}^{\dagger} \mathbf{G}_{\mathbf{x}}^{\dagger}-\mathbf{I}\right)\left(\mathbf{G}_{\mathbf{x}} \mathbf{B}-\mathbf{I}\right)+\alpha \mathbf{B}^{\dagger} \mathbf{B}
\end{aligned}
$$

where $\mathrm{SNR}_{m}=\alpha^{-1}=\sigma_{a}^{2} / \sigma_{n}^{2}$ is the signal-to-noise ratio (SNR) on the measure. The MSE expression specialized to the different filters is given below.

\section{A. Matched Filter}

As a first solution, we choose $\mathbf{B}$ as the filter matched to $\mathbf{G}_{\mathbf{x}}$. The MF is optimal when the collected samples are equally spaced, that is when the rows of $\sqrt{\beta} \mathbf{G}_{\mathbf{x}}$ are orthonormal vectors and $\sqrt{\beta} \mathbf{G}_{\mathbf{X}}$ is a unitary matrix (i.e., $\beta \mathbf{G}_{\mathbf{X}} \mathbf{G}_{\mathbf{X}}^{\dagger}=\mathbf{I}_{2 M+1}$ ). Thus, we choose

$$
\mathbf{B}^{\dagger}=\beta \mathbf{G}_{\mathbf{x}} .
$$

Recall that $\mathbf{G}_{\mathbf{X}}$ depends on the position vector $\mathbf{x}$ that, under Model A, coincides with the actual sensor positions. Indeed, in the absence of noise and for equally spaced sensors, the spectrum estimates perfectly match a, i.e., $\hat{\mathbf{a}}=\mathbf{B}^{\dagger} \mathbf{p}=\beta \mathbf{G}_{\mathbf{x}}^{\dagger} \mathbf{G}_{\mathbf{x}} \mathbf{a}=$ a. By replacing (13) in (12), we obtain

$$
\Psi_{\mathbf{x}}=\beta^{2} \mathbf{R}_{\mathbf{x}}^{2}+\mathbf{I}+(\alpha \beta-2) \beta \mathbf{R}_{\mathbf{x}}
$$

where $\mathbf{R}_{\mathbf{x}}=\mathbf{G}_{\mathbf{x}} \mathbf{G}_{\mathbf{x}}^{\dagger}$. From the definition in (9), the asymptotic MSE, averaged over the random vector $\mathbf{x}$, is given by

$$
\mathrm{MSE}_{\infty}=\beta^{2} \phi\left(\mathbf{R}_{\mathbf{x}}^{2}\right)+\phi(\mathbf{I})+(\alpha \beta-2) \beta \phi\left(\mathbf{R}_{\mathbf{x}}\right) .
$$

Notice that the second term on the right-hand side (RHS) reduces to 1 since $\phi(\mathbf{I})=1$. Applying (10), first with $g(x)=x^{2}$ and then with $g(x)=x$, we obtain

$$
\mathrm{MSE}_{\infty}=\mathbb{E}\left[\lambda^{2}\right]+1+(\alpha \beta-2) \mathbb{E}[\lambda]
$$

where $\lambda>0$ is the random variable with probability density function (pdf) $f_{\lambda, \beta}(x)$, distributed as the asymptotic eigenvalues of $\beta \mathbf{R}_{\mathbf{x}}$. In [30] it is shown that, for any positive integer $p, \mathbb{E}\left[\lambda^{p}\right]$ is a polynomial in $\beta$ of degree $p-1$. In particular, $\mathbb{E}[\lambda]=1$ and $\mathbb{E}\left[\lambda^{2}\right]=1+\beta$. We, therefore, obtain

$$
\mathrm{MSE}_{\infty}=\beta(\alpha+1)
$$

\section{B. ZF Filter}

The expression of the ZF filter for the system in (4) is

$$
\mathbf{B}^{\dagger}=\mathbf{R}_{\mathbf{x}}^{-1} \mathbf{G}_{\mathbf{x}} .
$$

Notice that, by its definition, the ZF filter does not exploit any information on the noise contribution (such as $\sigma_{n}^{2}$ ). However, this reconstruction technique takes into account the fact that the collected samples are not equally spaced and, hence, that $\sqrt{\beta} \mathbf{G}_{\mathbf{X}}$ is not a unitary matrix.
By using (17) in (12), the matrix $\Psi_{\mathrm{x}}$ becomes

$$
\Psi_{\mathbf{x}}=\alpha \mathbf{R}_{\mathbf{x}}^{-1} .
$$

Using (9) and applying (10) with $g(x)=x^{-1}$, the asymptotic MSE, averaged over the random vector $\mathbf{x}$, can be written as

$$
\mathrm{MSE}_{\infty}=\alpha \phi\left(\mathbf{R}_{\mathbf{x}}^{-1}\right)=\alpha \beta \mathbb{E}\left[\lambda^{-1}\right] .
$$

We make the following observations:

1) Since $\lambda^{-1}$ is a convex function, then $\mathbb{E}\left[\lambda^{-1}\right] \geq 1 / \mathbb{E}[\lambda]$. In [30] it is shown that $\mathbb{E}[\lambda]=1$, thus, $\operatorname{MSE}_{\infty} \geq \alpha \beta$.

2) We can empirically show that $\mathrm{MSE}_{\infty}=\alpha \beta \mathbb{E}\left[\lambda^{-1}\right]<$ $+\infty$ only for $0 \leq \beta<\beta^{\star}$, with $\beta^{\star} \approx 0.35$. Indeed, $\mathbb{E}\left[\lambda^{-1}\right]=\int_{0}^{+\infty} x^{-1} f_{\lambda, \beta}(x) \mathrm{d} x$. Through Monte Carlo simulation it has been observed that $f_{\lambda, \beta}(x) \propto x^{a(\beta)-1}$ for $x \ll 1$, where the exponent $a(\beta)$ is a decreasing function of $\beta$ for $0 \leq \beta<1$, and $a(\beta)=1$ for $\beta=\beta^{\star}$. Given that, for any positive constant $c$, we have

$$
\int_{0}^{c} \frac{1}{x} f_{\lambda, \beta}(x) \mathrm{d} x \propto \int_{0}^{c} x^{a(\beta)-2} \mathrm{~d} x
$$

where the integral on the RHS [and, therefore, (19)] does not diverge if and only if $a(\beta)>1$, that is $\beta<\beta^{\star}$. This observation gives us an empirical limit to the minimum number of sensors required to perform reliable reconstruction with the ZF filter.

\section{LMMSE Linear Filter}

A more efficient solution is to employ the filter $\mathbf{B}$ that provides the minimum MSE (LMMSE). By assuming that the SNR $\mathrm{SNR}_{m}$ is known to the sink and exploiting this information for the filter design, the expression of the LMMSE filter [31] for Model A in (4) is given by

$$
\mathbf{B}^{\dagger}=\left(\mathbf{R}_{\mathbf{x}}+\alpha \mathbf{I}\right)^{-1} \mathbf{G}_{\mathbf{x}} .
$$

We highlight that this reconstruction technique accounts for both the fact that the collected samples are non-uniformly spaced and the presence of the measurement noise.

Substituting (20) in (12), we obtain

$$
\Psi_{\mathbf{x}}=\alpha\left(\mathbf{R}_{\mathbf{x}}+\alpha \mathbf{I}\right)^{-1} .
$$

Using (10) with $g(x)=(x+\alpha \beta)^{-1}$, the asymptotic MSE is

$$
\mathrm{MSE}_{\infty}=\mathbb{E}\left[\frac{\alpha \beta}{\lambda+\alpha \beta}\right] .
$$

Note that

$$
\mathbb{E}\left[\frac{\alpha \beta}{\lambda+\alpha \beta}\right] \geq \frac{\alpha \beta}{\mathbb{E}[\lambda+\alpha \beta]}=\frac{\alpha \beta}{1+\alpha \beta} .
$$

Also, note that $\mathbb{E}[\alpha \beta /(\lambda+\alpha \beta)] \leq 1$, since $\lambda \geq 0$. Given that the LMMSE filter provides the minimum MSE, from (23) it turns out that, for a given $\beta, \alpha \beta /(1+\alpha \beta)$ is a lower bound for the performance of all linear reconstruction techniques. 


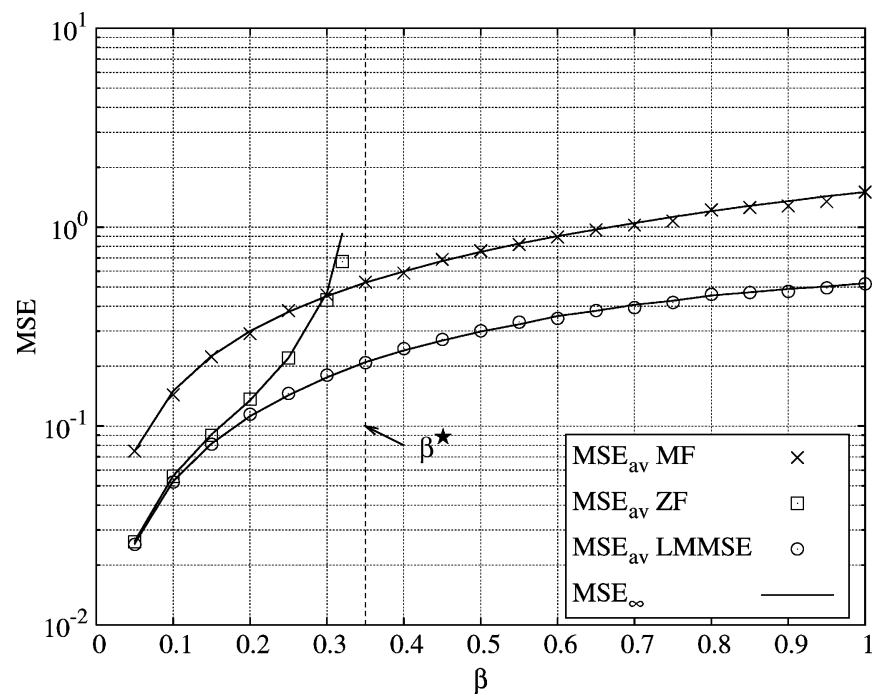

Fig. 1. MSE obtained through the MF, ZF, and the LMMSE filters, plotted versus $\beta$, for $M=40, \mathrm{SNR}_{m}=3 \mathrm{~dB}$ (i.e., $\alpha=1 / 2$ ).

\section{Results}

Fig. 1 compares the average MSE obtained using the MF, ZF, and LMMSE filters, when $\beta$ varies and $\alpha=1 / 2$. The points labeled by "MSE $E_{a v}$ MF," "MSE $E_{a v}$ ZF," and "MSE $E_{a v}$ LMMSE" have been obtained generating 100 realizations of the measures (4) with $M=40$, computing the estimates as in (5) and averaging the square error $\|\mathbf{a}-\hat{\mathbf{a}}\|^{2}$. These points are superimposed over the solid curves labeled by "MSE ${ }_{\infty}$," representing the asymptotic MSE and obtained evaluating (15), (19), and (22), respectively. Notice that computing closed form expressions for $\mathbb{E}[1 / \lambda]$ in (19) and $\mathbb{E}[\alpha \beta /(\lambda+\alpha \beta)]$ in (22) is still an open problem since a closed form expression of the distribution of $\lambda$ is unknown. Thus, for a given $\beta$, the value of these asymptotic expressions have been obtained pseudoanalytically, averaging over the eigenvalues $\lambda$ obtained by several realizations of the matrix $\beta \mathbf{R}_{\mathbf{x}}$, with $M=200$ which yields a very good approximation of the asymptotic case [30].

We observe an excellent agreement between the asymptotic analysis and the numerical results; this shows the validity of the asymptotic analysis even for small values of $M$. We also note that, for both filters, higher values of MSE are obtained as $\beta$ increases. Finally, the LMMSE filter provides the best performance, while the MSE of the ZF filter shows a vertical asymptote for $\beta=\beta^{\star}$, in agreement with the closed form analysis.

Fig. 2 shows the MSE versus $\mathrm{SNR}_{m}$, for $\beta=0.2$. The behavior of the asymptotic MSE is represented by the curves labeled by "MSE ${ }_{\infty}$ " while the average MSE obtained through numerical analysis is denoted by the label "MSE $\mathrm{av}_{\mathrm{av}}$." The curves have been obtained using the same procedure as for the results in Fig. 1, using $M=10$ for the computation of both $\mathrm{MSE}_{a v}$ and $\mathrm{MSE}_{\infty}$. Again, note the tight match between analytical and numerical results. For all techniques, the MSE decreases as the $\mathrm{SNR}_{m}$ increases. The MF however provides very poor performance, even for high $\mathrm{SNR}_{m}$. In particular, as $\mathrm{SNR}_{m}$ tends to infinity, it shows a horizontal asymptote with $\mathrm{MSE}_{\infty}=\beta$.

Besides linear filtering, another technique for estimating the spectrum $\mathbf{a}$ is based on interpolation. The idea is to interpo-

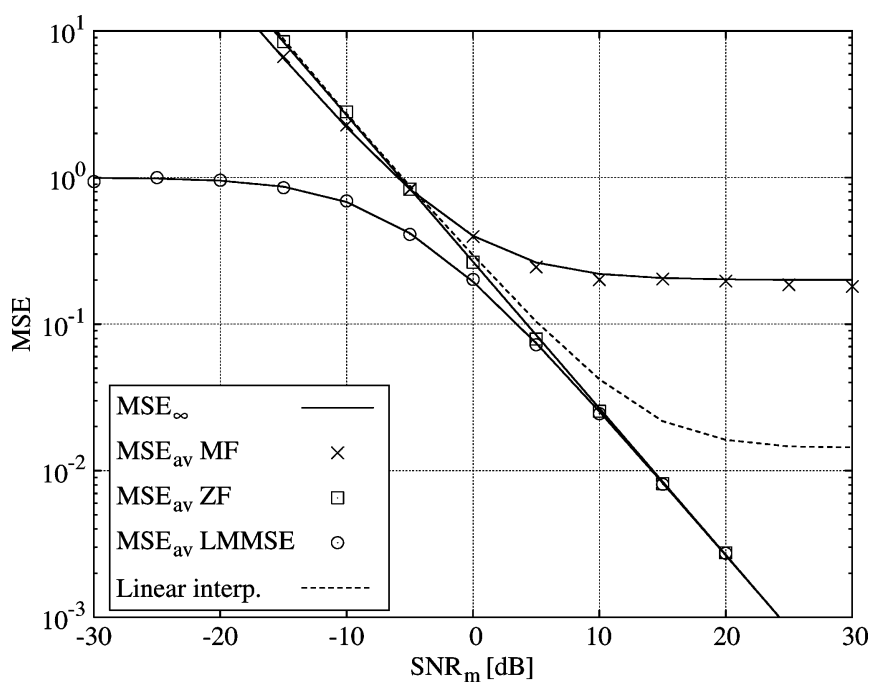

Fig. 2. MSE obtained with the MF, the ZF, and the LMMSE filters, plotted versus $\mathrm{SNR}_{m}$, for $\beta=0.2$ and $M=10$.

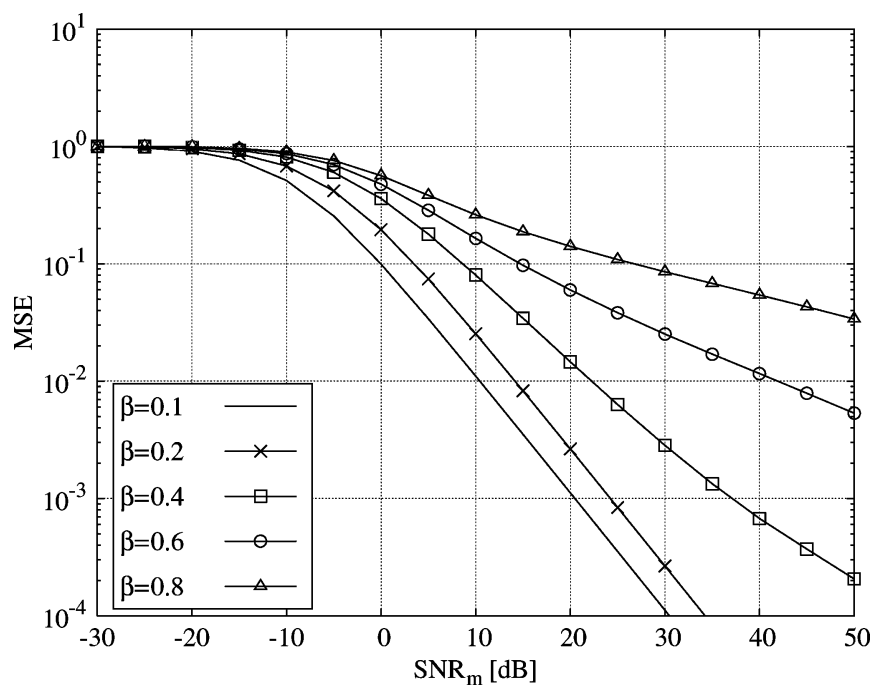

Fig. 3. MSE obtained through the LMMSE filter, plotted versus $\mathrm{SNR}_{m}$, for $\beta=0.1,0.2,0.4,0.6,0.8$, and $M=10$.

late the measures $\mathbf{p}$ to a regular sampling grid defined by the vector $\mathbf{x}^{\prime}$ where $x_{q}^{\prime}=(q-1) / r, q=1, \ldots, r$. The interpolated vector $\mathbf{p}^{\prime}$ is then multiplied by the matrix $\beta \mathbf{G}_{\mathbf{x}^{\prime}}$. Notice that in this case $\sqrt{\beta} \mathbf{G}_{\mathbf{x}^{\prime}}$ is unitary, i.e., $\beta \mathbf{G}_{\mathbf{x}^{\prime}} \mathbf{G}_{\mathbf{x}^{\prime}}^{\dagger}=\mathbf{I}$, since $\mathbf{x}^{\prime}$ represents an equally spaced sampling. In the plot, the dashed line labeled "Linear interp." shows the performance obtained using linear interpolation. The MSE has a horizontal asymptote for high $\mathrm{SNR}_{m}$. While it outperforms the MF, it clearly shows poor performance for high $\mathrm{SNR}_{m}$, compared to $\mathrm{ZF}$ and LMMSE techniques.

Fig. 3 presents the performance of the LMMSE filter obtained evaluating (22) for different values of $\beta$, as the $\mathrm{SNR}_{m}$ varies. In agreement with the results presented in Fig. 1, the performance of the LMMSE filter degrades as $\beta$ increases, while, as expected, it improves as the $\mathrm{SNR}_{m}$ increases.

Example 1: We need to estimate the number of sensors required to sample a field with $M=100$ harmonics. Each sensor provides samples with $\mathrm{SNR}_{m}=30 \mathrm{~dB}$. 
We choose to employ the LMMSE filter, which provides the best performance. Looking at Fig. 3, if we allow an $\mathrm{MSE}_{\mathrm{av}}$ of $3 \cdot 10^{-3}$, then we need $\beta=0.4$, i.e., $r=(2 M+1) / \beta \approx$ 500 sensors. By doubling the number of sensors $(\beta=0.2)$, $\mathrm{MSE}_{\mathrm{av}}$ drops to $3 \cdot 10^{-4}$.

\section{ANALYSIS OF MODEL B}

Here we consider the case of sensors with jittered positions and average position, $\hat{\mathbf{x}}$, known at the sink node. The true sensor location is: $\mathbf{x}=\hat{\mathbf{x}}+\boldsymbol{\delta}$, where $\delta$ is a random vector, as defined in Section II. The reconstruction algorithm employs the matrix $\mathbf{B}$, which is a function of the known average positions $\hat{\mathbf{x}}$. For any given $\hat{\mathbf{x}}$ and $\mathbf{B}$, similarly to (6), the MSE becomes

$$
\mathrm{MSE}_{\hat{\mathbf{x}}}=\frac{\mathbb{E}_{\mathbf{a}, \mathbf{n}, \delta}\left[\|\hat{\mathbf{a}}-\mathbf{a}\|^{2}\right]}{2 M+1}=\frac{\sigma_{a}^{2}}{2 M+1} \operatorname{Tr}\left\{\Psi_{\hat{\mathbf{x}}}\right\}
$$

where

$$
\begin{aligned}
\boldsymbol{\Psi}_{\hat{\mathbf{x}}} & =\frac{1}{\sigma_{a}^{2}} \mathbb{E}_{\mathbf{a}, \mathbf{n}, \boldsymbol{\delta}}\left[(\hat{\mathbf{a}}-\mathbf{a})(\hat{\mathbf{a}}-\mathbf{a})^{\dagger}\right] \\
& =\mathbb{E}_{\boldsymbol{\delta}}\left[\left(\mathbf{B}^{\dagger} \mathbf{G}_{\mathbf{x}}^{\dagger}-\mathbf{I}\right)\left(\mathbf{G}_{\mathbf{x}} \mathbf{B}-\mathbf{I}\right)+\alpha \mathbf{B}^{\dagger} \mathbf{B}\right] \\
& =\mathbf{B}^{\dagger}\left(\mathbb{E}_{\boldsymbol{\delta}}\left[\mathbf{G}_{\mathbf{x}}^{\dagger} \mathbf{G}_{\mathbf{x}}\right]+\alpha \mathbf{I}\right) \mathbf{B}-2 \Re\left\{\mathbb{E}_{\delta}\left[\mathbf{G}_{\mathbf{x}}\right] \mathbf{B}\right\}+\mathbf{I}
\end{aligned}
$$

with $\Re\{\cdot\}$ representing the real part of the argument.

To proceed further we need to compute the averages over the displacements $\boldsymbol{\delta}$, i.e., we need the expressions of $\underset{\boldsymbol{\delta}}{\mathbb{E}}\left[\mathbf{G}_{\mathbf{x}}\right]$ and $\underset{\delta}{\mathbb{E}}\left[\mathbf{G}_{\mathbf{x}}^{\dagger} \mathbf{G}_{\mathbf{x}}\right]$ as functions of $\mathbf{G}_{\hat{\mathbf{x}}}$, whose derivation is given in Appendix III. We have

$$
\underset{\delta}{\mathbb{E}}\left[G_{\mathbf{x}}\right]=\mathbf{C G}_{\hat{\mathbf{x}}}
$$

and

$$
\mathbb{E}_{\delta}\left[\mathbf{G}_{\mathbf{x}}^{\dagger} \mathbf{G}_{\mathbf{x}}\right]=\mathbf{G}_{\hat{\mathbf{x}}}^{\dagger} \mathbf{C}^{2} \mathbf{G}_{\hat{\mathbf{x}}}+\left(1-\frac{\operatorname{Tr}\left\{\mathbf{C}^{2}\right\}}{2 M+1}\right) \mathbf{I}
$$

where $\mathbf{C}$ is a $(2 M+1) \times(2 M+1)$ diagonal matrix with $(\mathbf{C})_{k k}=C_{\delta}(-\mathrm{j} 2 \pi k), k=-M, \ldots, M$, where $C_{\delta}(\cdot)$ is the characteristic function of the displacements. Under the assumption that $\delta$ has a zero mean Gaussian distribution, we have $(\mathbf{C})_{k k}=\exp \left(-2 \pi^{2} k^{2} \sigma_{\delta}^{2}\right), k=-M, \ldots, M$.

Using (26) and (27) in (25), we obtain

$$
\Psi_{\hat{\mathbf{x}}}=\mathbf{B}^{\dagger}\left(\mathbf{G}_{\hat{\mathbf{x}}}^{\dagger} \mathbf{C}^{2} \mathbf{G}_{\hat{\mathbf{x}}}+\gamma \mathbf{I}\right) \mathbf{B}-2 \Re\left\{\mathbf{C G}_{\hat{\mathbf{x}}} \mathbf{B}\right\}+\mathbf{I}
$$

where $\gamma=1+\alpha+\left(\operatorname{Tr}\left\{\mathbf{C}^{2}\right\} /(2 M+1)\right)$.

In the following, in the case of the LMMSE filter ${ }^{3}$ we first consider that the variance $\sigma_{\delta}^{2}$ of the sensor displacement is unknown at the sink and, hence, while running the reconstruction algorithm, the sink assumes the sensors to be fixed (i.e., $\boldsymbol{\delta}=\mathbf{0}$ ). Then, we consider that $\sigma_{\delta}^{2}$ is known and the reconstruction algorithm employs a filter that exploits such an information to minimize the MSE (this case is referred to as "LMMSE for known $\left.\sigma_{\delta}^{2} . "\right)$

${ }^{3}$ Recall that the MF and ZF techniques, by their definition, do not require any information on $\sigma_{n}^{2}$ and $\sigma_{\delta}^{2}$.
Finally, we remark that, while in Model A the filters used for signal reconstruction are functions of the matrix $\mathbf{G}_{\mathbf{X}}$ with $\mathrm{x}$ known to the sink, in Model $\mathrm{B}$ only the mean value of the sensor positions $\hat{\mathbf{X}}$ is known and, thus, the filters are computed using $\mathbf{G}_{\hat{\mathbf{x}}}$ instead of $\mathbf{G}_{\mathbf{x}}$.

\section{A. Matched Filter}

If the sink node employs the MF in (13) as function of $\hat{\mathbf{x}}$ (i.e., $\mathbf{B}^{\dagger}=\beta \mathbf{G}_{\hat{\mathbf{x}}}$ ), then, using (28), we obtain

$$
\boldsymbol{\Psi}_{\hat{\mathbf{x}}}=\beta^{2} \mathbf{R}_{\hat{\mathbf{x}}} \mathbf{C}^{2} \mathbf{R}_{\hat{\mathbf{x}}}+\gamma \beta^{2} \mathbf{R}_{\hat{\mathbf{x}}}-2 \beta \Re\left\{\mathbf{C R}_{\hat{\mathbf{x}}}\right\}+\mathbf{I} .
$$

This result holds for strictly positive $\sigma_{\delta}^{2}$. Note that, for $\sigma_{\delta}^{2}=0$ (no sensor displacement), we have $\mathbf{C}=\mathbf{I}$ and $\gamma=\alpha$; thus, (29) reduces to (14).

Equation (29) refers to the MSE obtained with a given vector $\hat{\mathbf{x}}$; we are now interested in deriving the asymptotic expression for the MSE. Note that (29) is a function of both $\mathbf{R}_{\hat{\mathbf{x}}}$ and $\mathbf{C}$, and contains terms of the form $\mathbf{C}^{p} g\left(\mathbf{R}_{\hat{\mathbf{x}}}\right)$ with $g(x)=1, x, x^{2}$ and $p=0,1,2$; also the matrix $\mathbf{R}_{\hat{\mathbf{x}}}$ depends on $M$ and $r$, while the matrix $\mathbf{C}$ depends on $M$ and $\sigma_{\delta}^{2}$. The definition of the asymptotic MSE in (8) refers to the case where the number of harmonics $M$ and the number of sensors $r$ grow to infinity with constant ratio $\beta$; if this is directly applied to (29), information losses may arise. Indeed, we have

$$
\begin{aligned}
\phi\left(\mathbf{C}^{p}\right) & =\lim _{\substack{M, r \rightarrow+\infty \\
\beta}} \frac{1}{2 M+1} \operatorname{Tr}\left\{\mathbf{C}^{p}\right\} \\
& =\lim _{M \rightarrow+\infty} \frac{1}{2 M+1} \sum_{k=-M}^{M} \mathrm{e}^{-2 p \pi^{2} k^{2} \sigma_{\delta}^{2}}=0
\end{aligned}
$$

and, thus, all terms depending on the matrix $\mathbf{C}$ would vanish regardless of the value of $\sigma_{\delta}^{2}$. On the contrary, in a realistic situation we expect to obtain high reconstruction quality when the standard deviation of the displacement $\left(\sigma_{\delta}\right)$ is smaller than or comparable to the average sensor separation $(1 / r)$, and a significant degradation of the reconstruction quality when $\sigma_{\delta}$ is much larger than the average sensor separation. To distinguish such different conditions, we define the $S N R$ on the displacement as

$$
\mathrm{SNR}_{x}=\frac{(1 / r)^{2}}{\sigma_{\delta}^{2}}=\frac{1}{\omega^{2}}
$$

where $\omega=\sigma_{\delta} r$. We then redefine the asymptotic MSE as the limit of the average MSE for $M, r \rightarrow+\infty$, with constant $\beta=$ $(2 M+1) / r$ and constant $\omega=\sigma_{\delta} r$. In this case

$$
\begin{aligned}
\phi\left(\mathbf{C}^{p}\right) & =\lim _{\substack{M, r \rightarrow+\infty \\
\sigma \delta \rightarrow \infty \\
\beta, \omega}} \frac{1}{2 M+1} \sum_{k=-M}^{M} \exp \left(-2 p \pi^{2} k^{2} \sigma_{\delta}^{2}\right) \\
& =\int_{-1 / 2}^{1 / 2} \exp \left(-2 p \pi^{2} z^{2} \beta^{2} \omega^{2}\right) \mathrm{d} z \\
& =\sqrt{\frac{\pi}{4}} \frac{\operatorname{erf}\left(\sqrt{\frac{p}{2}} \pi \beta \omega\right)}{\sqrt{\frac{p}{2}} \pi \beta \omega}=\nu\left(\sqrt{\frac{p}{2}} \beta \omega\right)
\end{aligned}
$$


where $\nu(x)=\sqrt{\pi / 4} \operatorname{erf}(\pi x) /(\pi x)$. Notice that $\nu(0)=1$ and $\lim _{x \rightarrow+\infty} \nu(x)=0$. Also, we have

$$
\phi(\gamma)=1+\alpha-\lim _{\substack{M, r \rightarrow+\infty \\ \delta, \rightarrow \infty \\ \beta, \omega}} \frac{\operatorname{Tr}\left\{\mathbf{C}^{2}\right\}}{2 M+1}=1+\alpha-\nu(\beta \omega) .
$$

Using the new definition and (29), the asymptotic expression of the MSE becomes

$$
\begin{aligned}
\operatorname{MSE}_{\infty}= & \operatorname{Tr}\left\{\Psi_{\hat{\mathbf{x}}}\right\} \\
= & \phi\left(\beta^{2} \mathbf{R}_{\hat{\mathbf{x}}} \mathbf{C}^{2} \mathbf{R}_{\hat{\mathbf{x}}}+\gamma \beta^{2} \mathbf{R}_{\hat{\mathbf{x}}}-2 \beta \Re\left\{\mathbf{C R}_{\hat{\mathbf{x}}}\right\}+\mathbf{I}\right) \\
= & \beta^{2} \phi\left(\mathbf{C}^{2} \mathbf{R}_{\hat{\mathbf{x}}}^{2}\right)+\beta^{2} \phi\left(\gamma \mathbf{R}_{\hat{\mathbf{x}}}\right)-2 \beta \phi\left(\mathbf{C R}_{\hat{\mathbf{x}}}\right)+1 \\
= & \beta^{2} \phi\left(\mathbf{C}^{2}\right) \phi\left(\mathbf{R}_{\hat{\mathbf{x}}}^{2}\right)+\beta^{2} \phi(\gamma) \phi\left(\mathbf{R}_{\hat{\mathbf{x}}}\right) \\
& -2 \beta \phi(\mathbf{C}) \phi\left(\mathbf{R}_{\hat{\mathbf{x}}}\right)+1 \\
= & \nu(\beta \omega) \mathbb{E}\left[\lambda^{2}\right]+\beta \phi(\gamma) \mathbb{E}[\lambda]-2 \nu(\beta \omega / \sqrt{2}) \mathbb{E}[\lambda]+1 \\
= & \nu(\beta \omega)(1+\beta)+\beta(1+\alpha-\nu(\beta \omega)) \\
& -2 \nu(\beta \omega / \sqrt{2})+1 \\
= & \beta(1+\alpha)+\nu(\beta \omega)-2 \nu(\beta \omega / \sqrt{2})+1
\end{aligned}
$$

Here we used the following facts:

- $\phi\left(\Re\left\{\mathbf{C R}_{\mathbf{x}}\right\}\right)=\phi\left(\mathbf{C R}_{\mathbf{x}}\right)$ since $\mathbf{R}_{\hat{\mathbf{x}}}$ is Hermitian and $\mathbf{C}$ is real and diagonal;

- $\operatorname{Tr}\left\{\mathbf{X}_{1} \mathbf{X}_{2}\right\}=\operatorname{Tr}\left\{\mathbf{X}_{2} \mathbf{X}_{1}\right\}$ for any square matrix $\mathbf{X}_{1}$ and $\mathbf{X}_{2}$;

- $\phi\left(\mathbf{C}^{p} \mathbf{R}_{\hat{\mathbf{x}}}^{q}\right)=\phi\left(\mathbf{C}^{p}\right) \phi\left(\mathbf{R}_{\hat{\mathbf{x}}}^{q}\right)$ for any positive integer $p$ and $q$. This assumption holds only if $\mathbf{C}$ and $\mathbf{R}_{\hat{\mathbf{x}}}$ are asymptotically free [32]. Since asymptotical freeness is in general very hard to prove, we will simply verify the validity of such assumption through numerical results.

- $\mathbb{E}\left[\lambda^{2}\right]=1+\beta$ and $\mathbb{E}[\lambda]=1$ (see [30]);

Equation (33) reduces to (16) for $\omega=0$, while it reduces to $\mathrm{MSE}_{\infty}=1+\beta(1+\alpha)$ for $\omega=+\infty$.

\section{B. ZF Filter}

In this case the sink node employs the ZF filter in (17) but, knowing only the average value of the sensor positions, the filter results to be a function of $\hat{\mathbf{x}}: \mathbf{B}^{\dagger}=\mathbf{R}_{\hat{\mathbf{x}}}^{-1} \mathbf{G}_{\hat{\mathbf{x}}}$, and the matrix $\boldsymbol{\Psi}_{\hat{\mathbf{x}}}$ can be written as

$$
\boldsymbol{\Psi}_{\hat{\mathbf{x}}}=\gamma \mathbf{R}_{\hat{\mathbf{x}}}^{-1}+(\mathbf{C}-\mathbf{I})^{2} .
$$

We observe that, when $\sigma_{\delta}^{2}=0$ (no sensor displacement, we have $\mathbf{C}=\mathbf{I}$ and $\gamma=\alpha$, thus, (34) reduces to (18).

Using (31) and (32), the asymptotic MSE is

$$
\begin{aligned}
\operatorname{MSE}_{\infty}= & \phi\left(\gamma \mathbf{R}_{\hat{\mathbf{x}}}^{-1}+(\mathbf{C}-\mathbf{I})^{2}\right) \\
= & \beta(1+\alpha-\nu(\beta \omega)) \mathbb{E}\left[\frac{1}{\lambda}\right]+1+\nu(\beta \omega) \\
& -2 \nu(\beta \omega / \sqrt{2}) .
\end{aligned}
$$

Equation (35) reduces to (19) for $\omega=0$, while it reduces to $\mathrm{MSE}_{\infty}=1+\beta(1+\alpha) \mathbb{E}[1 / \lambda]$ for $\omega=+\infty$.

\section{LMMSE Filter Neglecting $\sigma_{\delta}^{2}$}

If the sink employs the filter in (20) computed using $\hat{\mathbf{x}}$ (i.e., $\mathbf{B}^{\dagger}=\mathbf{A}_{\hat{\mathbf{x}}}^{-1} \mathbf{G}_{\hat{\mathbf{x}}}$, where $\left.\mathbf{A}_{\hat{\mathbf{x}}}=\mathbf{R}_{\hat{\mathbf{x}}}+\alpha \mathbf{I}\right)$, then the matrix $\boldsymbol{\Psi}_{\hat{\mathbf{x}}}$ in (28) becomes:

$$
\Psi_{\hat{\mathbf{x}}}=\mathbf{A}_{\hat{\mathbf{x}}}^{-1} \mathbf{R}_{\hat{\mathbf{x}}}\left(\mathbf{C}^{2} \mathbf{R}_{\hat{\mathbf{x}}}+\gamma \mathbf{I}\right) \mathbf{A}_{\hat{\mathbf{x}}}^{-1}-2 \Re\left\{\mathbf{C R}_{\hat{\mathbf{x}}} \mathbf{A}_{\hat{\mathbf{x}}}^{-1}\right\}+\mathbf{I}
$$

For $\sigma_{\delta}^{2}=0$ (i.e., $\mathbf{C}=\mathbf{I}$ and $\gamma=\alpha$ ), (36) reduces to (21).

Using the properties described in Section VI-A the asymptotic MSE is:

$$
\begin{aligned}
& \operatorname{MSE}_{\infty}=1+(\nu(\beta \omega)-2 \nu(\beta \omega / \sqrt{2})) \mathbb{E}\left[\frac{\lambda^{2}}{(\lambda+\alpha \beta)^{2}}\right] \\
& \quad+\beta(1+\alpha-\nu(\beta \omega)-2 \alpha \nu(\beta \omega / \sqrt{2})) \mathbb{E}\left[\frac{\lambda}{(\lambda+\alpha \beta)^{2}}\right]
\end{aligned}
$$

Equation (37) reduces to (22) for $\omega=0$, while it becomes: $\mathrm{MSE}_{\infty}=1+\beta(1+\alpha) \mathbb{E}\left[\lambda /(\lambda+\alpha \beta)^{2}\right]$ for $\omega=+\infty$.

\section{LMMSE Filter for Known $\sigma_{\delta}^{2}$}

We now consider the linear LMMSE filter optimized for the case where $\sigma_{\delta}^{2}$ is known at the sink. We find the optimal $\mathbf{B}$ minimizing $\operatorname{Tr}\left\{\Psi_{\hat{x}}\right\}$; that is, we null the derivative of (24) with respect to $\mathbf{B}$. We employ the following properties that hold for any square matrix $\mathbf{X}[34]$ :

$$
\begin{aligned}
\frac{\partial}{\partial \mathbf{B}} \Re \operatorname{Tr}\{\mathbf{X B}\} & =\mathbf{X}^{\dagger} \\
\frac{\partial}{\partial \mathbf{B}} \operatorname{Tr}\left\{\mathbf{B}^{\dagger} \mathbf{X B}\right\} & =2 \mathbf{X B} \quad \text { if } \mathbf{X}=\mathbf{X}^{\dagger}
\end{aligned}
$$

Then, we have

$$
\frac{\partial \mathrm{MSE}_{\hat{\mathbf{x}}}(\mathbf{B})}{\partial \mathbf{B}}=\frac{2 \sigma_{a}^{2}\left(\mathbf{G}_{\hat{\mathbf{x}}}^{\dagger} \mathbf{C}^{2} \mathbf{G}_{\hat{\mathbf{x}}}+\gamma \mathbf{I}\right) \mathbf{B}}{2 M+1}-\frac{2 \sigma_{a}^{2} \mathbf{G}_{\hat{\mathbf{x}}}^{\dagger} \mathbf{C}}{2 M+1}=\mathbf{0} .
$$

Solving for $\mathbf{B}$, we obtain the expression of the LMMSE filter

$$
\mathbf{B}^{\dagger}=\left(\mathbf{C R}_{\hat{\mathbf{x}}} \mathbf{C}+\gamma \mathbf{I}\right)^{-1} \mathbf{C G}_{\hat{\mathbf{x}}} .
$$

Substituting (38) into (28), we have

$$
\boldsymbol{\Psi}_{\hat{\mathbf{x}}}=\gamma\left(\mathbf{C R}_{\hat{\mathbf{x}}} \mathbf{C}+\gamma \mathbf{I}\right)^{-1} .
$$

In this case an explicit expression of $\mathrm{MSE}_{\infty}$ is hard to obtain. However, we were able to find the following lower bound that turns out to be very tight, as shown by the results presented in the following section:

$$
\begin{aligned}
\operatorname{MSE}_{\infty} & =\phi\left(\gamma\left(\mathbf{C R}_{\hat{\mathbf{x}}} \mathbf{C}+\gamma \mathbf{I}\right)^{-1}\right) \\
& \geq \frac{1}{\phi\left(\frac{1}{\gamma}\left(\mathbf{C R}_{\hat{\mathbf{x}}} \mathbf{C}+\gamma \mathbf{I}\right)\right)} \\
& =\frac{\phi(\gamma)}{\phi\left(\mathbf{C}^{2} \mathbf{R}_{\hat{\mathbf{x}}}\right)+\phi(\gamma)} \\
& =\beta \frac{1+\alpha-\nu(\beta \omega)}{\beta(1+\alpha)+\nu(\beta \omega)(1-\beta)}
\end{aligned}
$$

where, to derive the last expression, we exploited (31), (32), (10), and the fact that $\mathbb{E}[\lambda]=1$. 


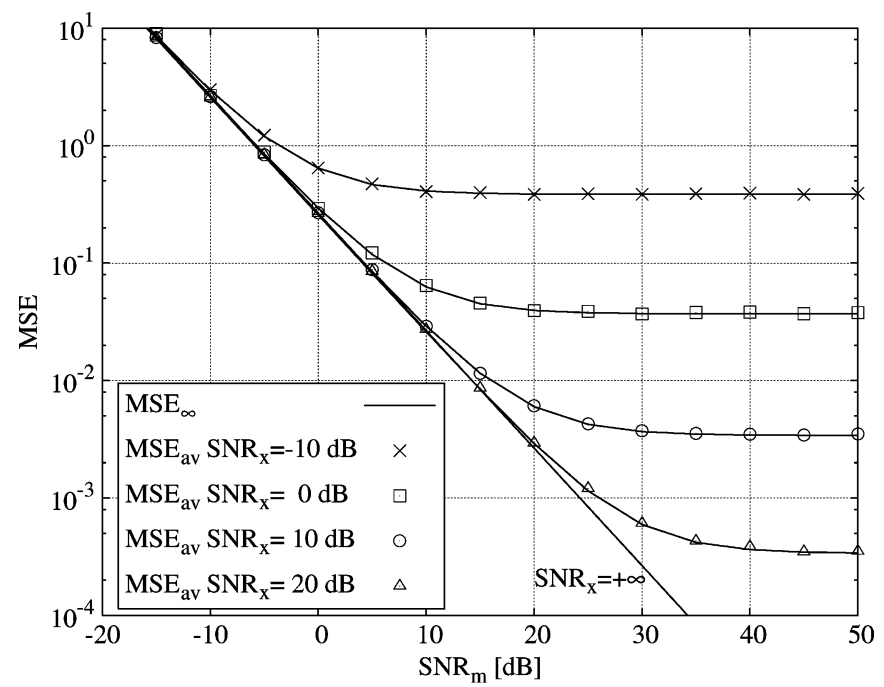

Fig. 4. Performance of the ZF filter for $\beta=0.2$ and $M=10$, when $\sigma_{\delta}^{2}$ is neglected.

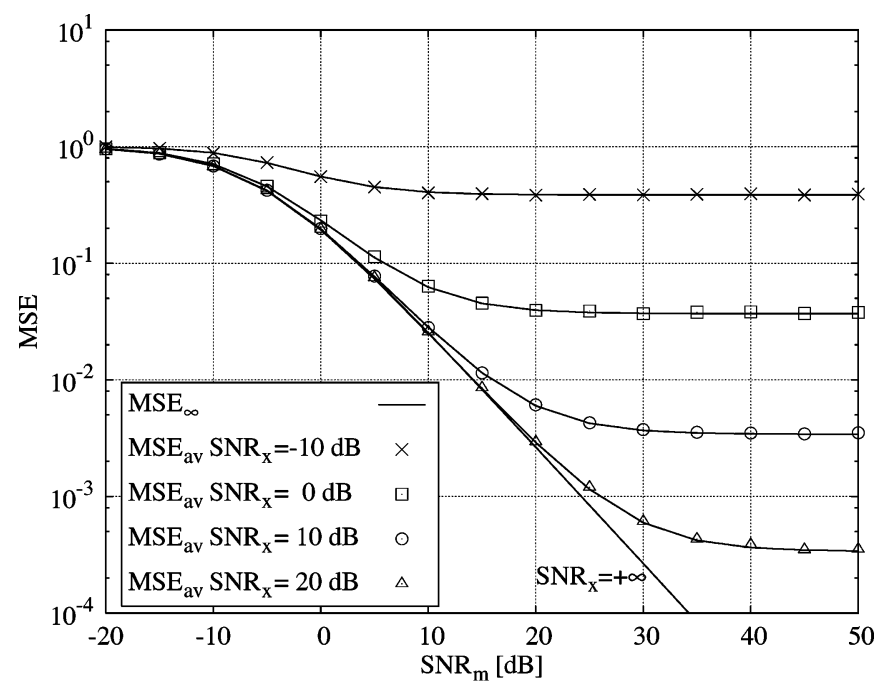

Fig. 5. Performance of the LMMSE filter (20) with $\mathbf{x}$ replaced by $\hat{\mathbf{x}}$ versus $\mathrm{SNR}_{m}$, for $\beta=0.2$ and $M=10$, when $\sigma_{\delta}^{2}$ is neglected

\section{E. Results}

We now show the performance of the filters analyzed under Model B. Regarding the ZF filter (17), Fig. 4 compares the asymptotic MSE evaluated through (35) (represented by solid lines and labeled by "MSE ${ }_{\infty}$ ") against the average MSE (represented by points and labeled by "MSE ${ }_{\mathrm{av}}$ "). The $\mathrm{MSE}_{\mathrm{av}}$ is obtained by generating 100 realizations of the measures as in (4), with $M=10$, computing the estimates as in (5) and averaging the square error $\|\mathbf{a}-\hat{\mathbf{a}}\|^{2}$. The MSE is shown in the log scale plotted versus $\mathrm{SNR}_{m}$, for $\beta=0.2$.

Similarly, Fig. 5 presents the performance of the LMMSE filter (20) with $\mathbf{x}$ replaced by $\hat{\mathbf{x}}$. Here the curves labeled by "MSE ${ }_{\infty}$ ", generated by evaluating (37), and the points in the plot, labeled by "MSE $\mathrm{av}_{\mathrm{av}}$ ", have been obtained as for Fig. 4.

In both the plots the solid line labeled by "SNR $=+\infty$ " refers to the case where $\omega=0$, i.e., $\delta=0$, and corresponds to the performance of Model A under the same conditions.

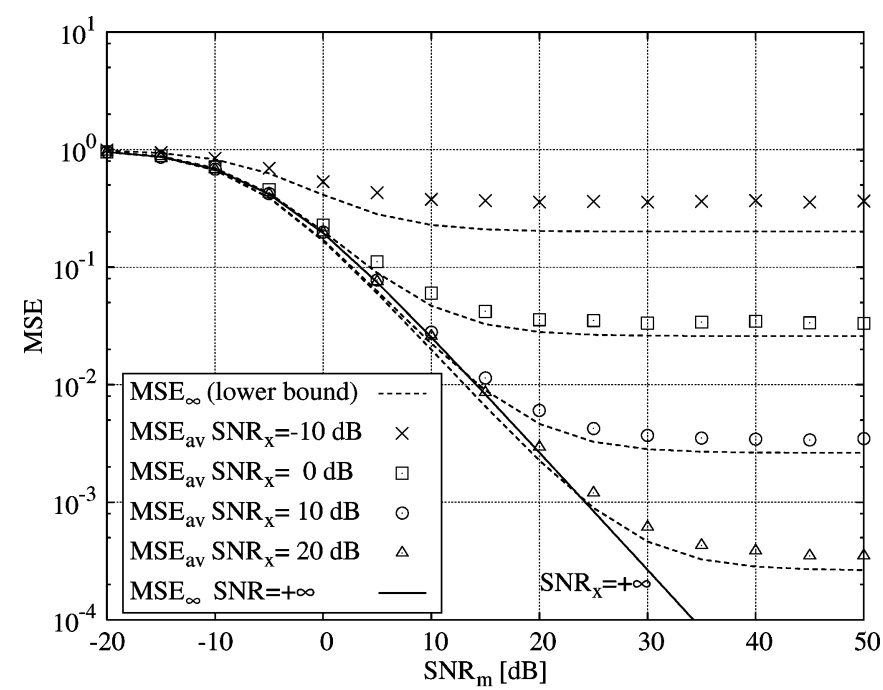

Fig. 6. Performance of the LMMSE filter (38) with perfect knowledge of $\sigma_{\delta}^{2}$ versus $\mathrm{SNR}_{m}$, for $\beta=0.2$ and $M=10$.

The excellent match between the asymptotic results and the numerical simulation confirms the validity of the asymptotic analysis as an effective tool to characterize the performance of the reconstruction techniques.

Also, comparing Figs. 4 and 5, we observe that the performances of the ZF and the LMMSE filters are similar for $\mathrm{SNR}_{m}>10 \mathrm{~dB}$, for any value of $\mathrm{SNR}_{x}$. While, for lower $\mathrm{SNR}_{m}$, the LMMSE filter outperforms the ZF filter.

Fig. 6 compares the performance of the LMMSE filter (38), which has knowledge of $\sigma_{\delta}^{2}$, with its lower bound (40) (dashed lines), as $\mathrm{SNR}_{m}$ varies. We consider $\beta=0.2$ and different values of $\mathrm{SNR}_{x}$. Notice that the lower bound is very tight, especially for high values of $\mathrm{SNR}_{x}$. The points in the plot, labeled by "MSE $\mathrm{av}_{\mathrm{av}}$ ", have been obtained as for Fig. 4, using $M=10$. Here, as in Fig. 5, the plot shows the case $\mathrm{SNR}_{x}=+\infty$ (solid line), which corresponds to the performance of the LMMSE filter for signal model A. Indeed, for $\mathrm{SNR}_{x}=\infty$ (i.e., $\sigma_{\delta}^{2}=0$ and $\boldsymbol{\delta}=0$ ), we have $\mathbf{C}=\mathbf{I}$ and $\gamma=\alpha$, and (39) simplifies to $(21)$.

Fig. 7 compares the performance of the LMMSE filter (20) (with $\mathrm{x}$ replaced by $\hat{\mathbf{x}}$ ), labeled by "LMMSE" (solid lines), and of the LMMSE filter (38), labeled by "LMMSE $\sigma_{\delta}^{2}$ " (dashed lines), for the same parameter setting as in Fig. 6. For the considered value of $\beta(\beta=0.2)$, the filter in (38) outperforms the simpler filter (20) for any value of $\mathrm{SNR}_{m}$ and $\mathrm{SNR}_{x}$, but the performance gain is always negligible.

Similarly to Fig. 5, Fig. 8 presents the results obtained through the LMMSE filter (20) (with $\mathrm{x}$ replaced by $\hat{\mathbf{x}}$ ), which neglects $\sigma_{\delta}^{2}$. Its performance has been obtained by evaluating (37) for $\mathrm{SNR}_{x}=10 \mathrm{~dB}$ (dashed lines) and $\mathrm{SNR}_{x}=20 \mathrm{~dB}$ (solid lines), and for $\beta=0.1,0.2,0.4$. While the $\mathrm{MSE}_{\infty}$ of the LMMSE filter (20) always tends to 1 for small values of $\mathrm{SNR}_{m}$ (i.e., large values of $\alpha$ ), for high $\operatorname{SNR}_{m}$ (i.e., low $\alpha$ ) its behavior depends on $\beta$. Indeed the term $\left.\mathbb{E}\left[\lambda /(\lambda+\alpha \beta)^{2}\right)\right]$ on the RHS of (37) reduces to $\mathbb{E}[1 / \lambda]$ for $\alpha \rightarrow 0$. As explained in Section V-B, $\mathbb{E}[1 / \lambda]$ diverges for $\beta>\beta^{\star} \approx 0.35$ and so the MSE (see the lines with o markers in the plot). This behavior, due to the model mismatch, is more evident as $\beta$ increases and 


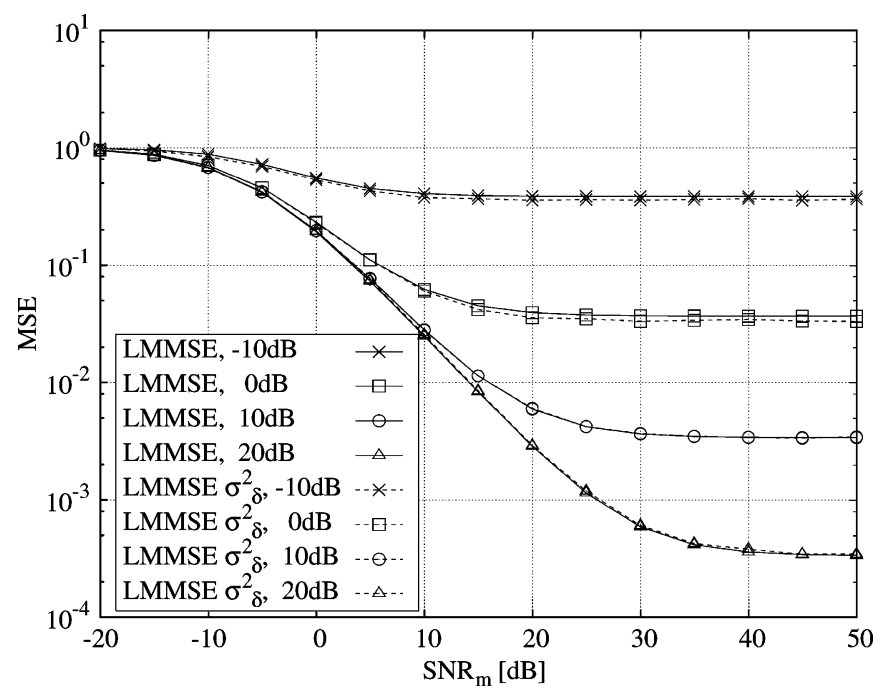

Fig. 7. Performance comparison of the LMMSE filter neglecting $\sigma_{\delta}^{2}$ (20) (with $\mathbf{x}$ replaced by $\hat{\mathbf{x}}$ ) against the LMMSE filter with perfect knowledge of $\sigma_{\delta}^{2}$, as $\mathrm{SNR}_{m}$ varies and for $\beta=0.2$ and $M=10$.

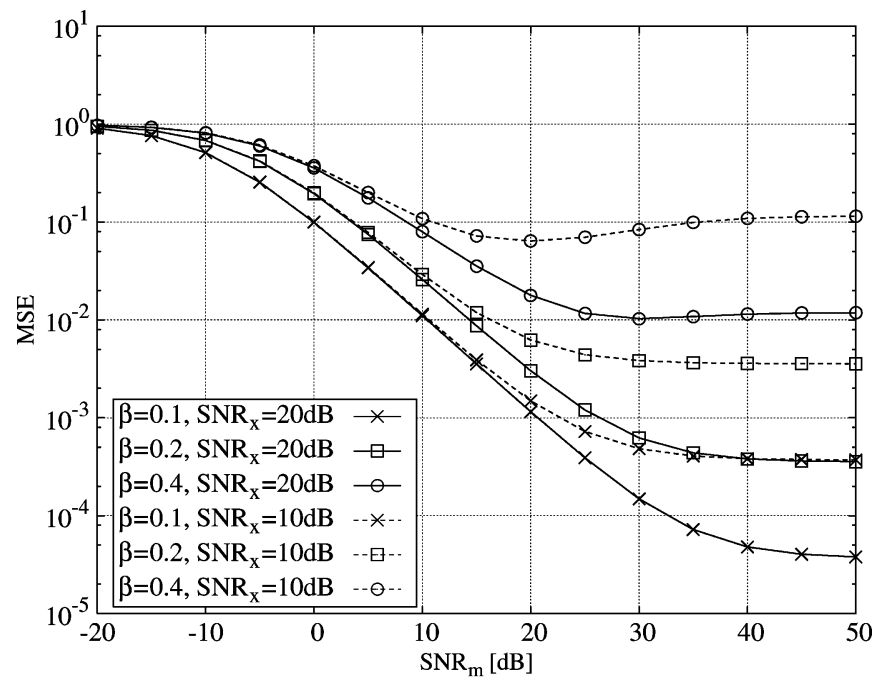

Fig. 8. Performance of the LMMSE filter when $\sigma_{\delta}^{2}$ is neglected as $\mathrm{SNR}_{m}$ varies, for $\mathrm{SNR}_{x}=10,20 \mathrm{~dB}$, and $\beta=0.1,0.2,0.4$, and $M=10$.

the MSE is large, for any $\mathrm{SNR}_{m}$. These results, however, are of no interest from the application point of view since a system characterized by such poor performance is not working.

Finally, Fig. 9 compares the performance of the LMMSE filter in (38) labeled by "LMMSE $\sigma_{\delta}^{2}$ " (dashed lines) and the performance of the LMMSE filter (20), labeled by "LMMSE" (solid lines), for $\mathrm{SNR}_{x}=10 \mathrm{~dB}$. In general, the filter in (38) performs always better than filter (20). In particular, for $\beta<$ $\beta^{\star}$ the two filters show very similar performance, while, when $\beta>\beta^{\star}$, the filter in (38) does not follow the performance of filter (20) for high $\mathrm{SNR}_{m}$. This is shown in Fig. 9, where, for $\beta>0.35$ and high values of $\mathrm{SNR}_{m}$, the advantage of exploiting the knowledge of $\sigma_{\delta}^{2}$ becomes evident.

Example 2: Consider $r=1000$ water buoys equipped with sensors, providing measures with $\mathrm{SNR}_{m}=30 \mathrm{~dB}$. Buoys are moving but the variance $\sigma_{\delta}^{2}=10^{-7}$ is unknown. We need to estimate the maximum number of harmonics of the field that

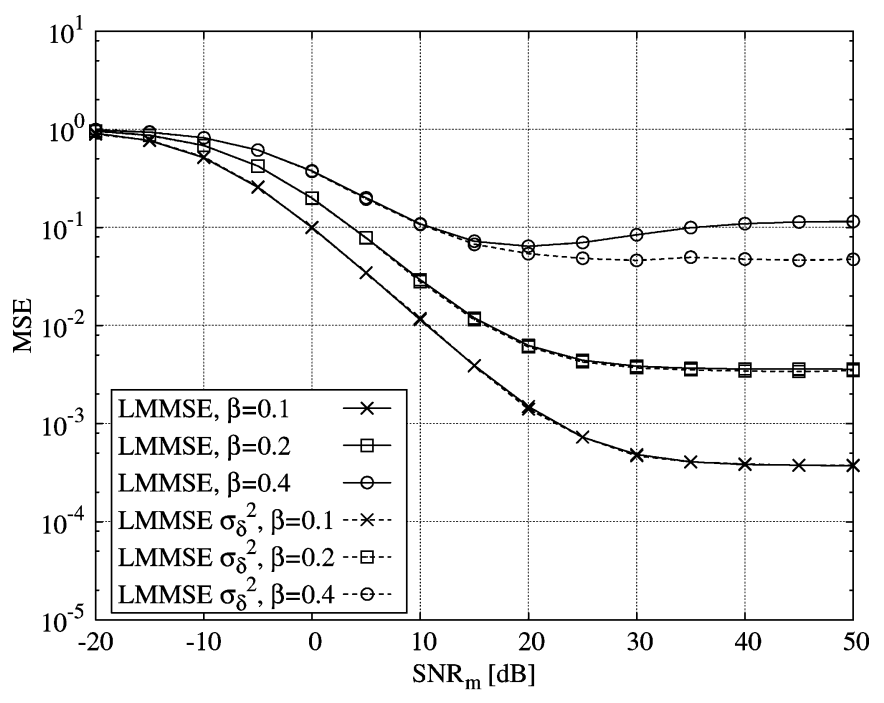

Fig. 9. Comparison of the LMMSE filter neglecting $\sigma_{\delta}^{2}$ against the LMMSE filter with perfect knowledge of $\sigma_{\delta}^{2}$, for $\mathrm{SNR}_{x}=10 \mathrm{~dB}$, and $M=10$.

the network can sample and reconstruct with an average MSE lower than $510^{-3}$.

Since $\mathrm{SNR}_{m}$ is known to the reconstruction algorithm while $\sigma_{\delta}^{2}$ is not, we employ the LMMSE filter in Section VI-C. We have: $\mathrm{SNR}_{x}=1 /\left(\sigma_{\delta}^{2} r^{2}\right)=10$. Looking at Fig. 9, we notice that, for $\mathrm{SNR}_{m}=30 \mathrm{~dB}$, values of $\mathrm{MSE}_{\mathrm{av}}$ lower than $510^{-3}$ can be obtained only for $\beta<0.2$. The maximum number of harmonics is then $M=(r \beta-1) / 2 \approx 100$.

Example 3: Consider a network of sensors with jittered positions characterized by $\beta=0.4$ and $\mathrm{SNR}_{x}=10 \mathrm{~dB}$, and assume that these values are known to the reconstruction algorithm. We want to determine which type of sensor devices should be used in order to minimize the $\mathrm{MSE}_{\mathrm{av}}$. In other words, we ask ourselves how accurate the sensor measurements need to be (clearly, more expensive devices provide a higher $\mathrm{SNR}_{m}$ ).

Since $\mathrm{SNR}_{x}=10 \mathrm{~dB}$ is known to the reconstruction algorithm, we can employ the LMMSE filter given in (38). Looking at Fig. 9, we notice that the performance of the filter for $\beta=0.4$ shows a horizontal asymptote corresponding to an average MSE of $5 \cdot 10^{-2}$. Thus, an $\mathrm{SNR}_{m}=25 \mathrm{~dB}$ is enough to achieve the best performance.

\section{SUMMARY OF RESUlTS}

Our main results for the system models A and B are as follows.

Model A (fixed sensors and noisy measures):

- for a given $\beta$, the MSE provided by any of the reconstruction techniques is lower bounded by $\alpha \beta /(1+\alpha \beta)$ and worsen with increasing $\beta$ (i.e., the ratio of the number of harmonics to the number of sampling sensors). The MF in (13) is the only filter which does not require matrix inversion, however it provides poor performance in all of the considered cases;

- the ZF filter provides high quality performance only for high $\mathrm{SNR}_{m}$ (namely, $\mathrm{SNR}_{m}>10 \mathrm{~dB}$ ) and $\beta<0.35$;

- the LMMSE filter, instead, gives good performances for moderate values of $\mathrm{SNR}_{m}$ and $\beta<1$. 
Model B (sensors with jittered positions and noisy measures):

- for a given $\beta$, the MSE provided by any of the reconstruction techniques is lower bounded by (40);

- the performance of all reconstruction techniques worsen with increasing $\beta$ and decreasing $\mathrm{SNR}_{x}$;

- the advantage of exploiting the knowledge of $\mathrm{SNR}_{x}$ in the filter design is negligible for low $\beta$ and low $\mathrm{SNR}_{m}$, while it is of fundamental importance to obtain a high quality reconstruction for $\beta>0.35$ and large values of $\mathrm{SNR}_{m}$.

\section{CONCLUSIONS}

We addressed the problem of reconstructing band-limited fields from measurements taken by irregularly deployed sensors, and we studied the effects of noisy measures and jittered sensors positions on the reconstruction quality. We analytically derived the performance of several linear filters in terms of the MSE of the field estimates. We also studied the asymptotic MSE, obtained as the number of harmonics and the number of sensors grow to infinity, while their ratio $\beta$ is kept constant. We found that the asymptotic analysis is an effective tool to characterize the performance of the reconstruction techniques, even for a small number of sensors, and we investigated the impact of the parameter $\beta$ on the system performance. In [30] we observed that random sampling without any type of noise would require more than twice the sampling rate of minimum regular sampling $(\beta=1)$ to get a reliable reconstruction (without ill conditioning problems) with high probability. The number of sensors further increases $(\beta<0.2)$ compared to regular sampling when measurement noise (Model A) and sensors position jitter (Model B) are present.

The main novelty of this work is the probabilistic approach to the performance analysis of linear reconstruction algorithms. A theoretical analysis was possible thanks to the linearity of the reconstruction methods that we adopted.

\section{APPENDIX I}

PROOF OF (10)

Let us consider an analytic function $g(\cdot)$ in $\mathbb{R}^{+}$. Let $\mathbf{X}=$ $\mathbf{U} \boldsymbol{\Lambda} \mathbf{U}^{\dagger}$ be a positive definite Hermitian $n \times n$ matrix, where $\mathbf{U}$ is the eigenvectors matrix of $\mathbf{X}$ and $\boldsymbol{\Lambda}$ is a diagonal matrix containing the eigenvalues of $\mathbf{X}$. Then, by using the result for symmetric matrices in [35, Ch. 6] combined with the result in [36, p. 481], we have

$$
\begin{aligned}
\phi(g(\mathbf{X})) & =\phi\left(g\left(\mathbf{U} \boldsymbol{\Lambda} \mathbf{U}^{\dagger}\right)\right)=\phi\left(\mathbf{U} g(\boldsymbol{\Lambda}) \mathbf{U}^{\dagger}\right) \\
& =\lim _{n \rightarrow \infty} \frac{1}{n} \mathbb{E}\left[\operatorname{Tr}\left\{\mathbf{U} g(\boldsymbol{\Lambda}) \mathbf{U}^{\dagger}\right\}\right] \\
& =\lim _{n \rightarrow \infty} \frac{1}{n} \mathbb{E}[\operatorname{Tr}\{g(\boldsymbol{\Lambda})\}] \\
& =\mathbb{E}[g(\xi)] .
\end{aligned}
$$

The last equality holds since, asymptotically, the eigenvalues of $\mathbf{X}$ are identically distributed.
APPENDIX II

PROOF OF LEMMA IV.1

Using (11), the $k q$ th entry of $\mathbf{G}_{\mathbf{X}}$ is

$$
\begin{aligned}
\left(\mathbf{G}_{\mathbf{x}}\right)_{k q} & =\sum_{n=0}^{\infty} \frac{1}{n !}\left(\mathbf{W}^{n} \mathbf{G}_{\hat{\mathbf{x}}} \Delta^{n}\right)_{k q} \\
& =\sum_{n=0}^{\infty} \frac{1}{n !} \sum_{h} \sum_{j}\left(\mathbf{W}^{n}\right)_{k h}\left(\mathbf{G}_{\hat{\mathbf{x}}}\right)_{h j}(\boldsymbol{\Delta})_{j q}^{n} \\
& =\sum_{n=0}^{\infty} \frac{1}{n !}\left(\mathbf{W}^{n}\right)_{k k}\left(\mathbf{G}_{\hat{\mathbf{x}}}\right)_{k q}\left(\boldsymbol{\Delta}^{n}\right)_{q q} \\
& =\exp \left((\mathbf{W})_{k k}(\boldsymbol{\Delta})_{q q}\right)\left(\mathbf{G}_{\hat{\mathbf{x}}}\right)_{k q} \\
& =\exp \left(-\mathrm{j} 2 \pi k \delta_{q}\right)\left(\mathbf{G}_{\hat{\mathbf{x}}}\right)_{k q} \\
& =\frac{1}{\sqrt{2 M+1}} \exp \left(-\mathrm{j} 2 \pi k x_{q}\right)
\end{aligned}
$$

which matches its definition.

\section{APPENDIX III$$
\text { COMPUTATION OF } \mathbb{E}\left[\mathbf{G}_{\mathbf{x}}\right] \text { AND } \mathbb{E}\left[\mathbf{G}_{\mathbf{x}}^{\dagger} \mathbf{G}_{\mathbf{x}}\right]
$$

We derive here the expressions of $\mathbb{E}\left[\mathbf{G}_{\mathbf{x}}\right]$ and $\mathbb{E}\left[\mathbf{G}_{\mathbf{x}}^{\dagger} \mathbf{G}_{\mathbf{x}}\right]$ as functions of $\mathbf{G}_{\hat{\mathbf{x}}}$.

a) Computation of $\mathbb{E}\left[\mathbf{G}_{\mathbf{X}}\right]$ : Using Lemma IV.1, we have

$$
\mathbb{E}\left[\mathbf{G}_{\mathbf{x}}\right]=\mathbb{E}\left[\sum_{n=0}^{\infty} \frac{1}{n !} \mathbf{W}^{n} \mathbf{G}_{\hat{\mathbf{x}}} \boldsymbol{\Delta}^{n}\right]=\sum_{n=0}^{\infty} \frac{1}{n !} \mathbf{W}^{n} \mathbf{G}_{\hat{\mathbf{x}}} \mathbb{E}\left[\boldsymbol{\Delta}^{n}\right] .
$$

The average of $\boldsymbol{\Delta}^{n}$ is given by $\mathbb{E}\left[\boldsymbol{\Delta}^{n}\right]=\int_{-\infty}^{+\infty} x^{n} f_{\delta}(x) \mathrm{d} x \mathbf{I}_{r}=$ $\mu^{(n)} \mathbf{I}_{r}$, where $\mathbf{I}_{r}$ is the $r \times r$ identity matrix and $\mu^{(n)}$ is the $n$th moment of $\delta$. Hence

$$
\begin{aligned}
\mathbb{E}\left[\mathbf{G}_{\mathbf{x}}\right] & =\sum_{n=0}^{\infty} \frac{1}{n !} \mathbf{W}^{n} \mathbf{G}_{\hat{\mathbf{x}}} \int_{-\infty}^{+\infty} x^{n} f_{\delta}(x) \mathrm{d} x \\
& =\int_{-\infty}^{+\infty} \sum_{n=0}^{\infty} \frac{x^{n}}{n !} \mathbf{W}^{n} f_{\delta}(x) \mathrm{d} x \mathbf{G}_{\hat{\mathbf{x}}} \\
& =\int_{-\infty}^{+\infty} \exp (x \mathbf{W}) f_{\delta}(x) \mathrm{d} x \mathbf{G}_{\hat{\mathbf{x}}}=\mathbf{C G}_{\hat{\mathbf{x}}}
\end{aligned}
$$

where ${ }^{4}$

$$
\begin{aligned}
\mathbf{C} & =\int_{-\infty}^{+\infty} \sum_{n=0}^{\infty} \frac{x^{n}}{n !} \mathbf{W}^{n} f_{\delta}(x) \mathrm{d} x \\
& =\sum_{n=0}^{\infty} \frac{\mu^{(n)}}{n !} \mathbf{W}^{n}=\int_{-\infty}^{+\infty} \exp (x \mathbf{W}) f_{\delta}(x) \mathrm{d} x
\end{aligned}
$$

${ }^{4}$ Let $\mathbf{A}=\operatorname{diag}\left(a_{1}, \ldots, a_{n}\right)$ be a diagonal $n \times n$ matrix. The exponential of $\mathbf{A}$, denoted by $\exp (\mathbf{A})$, is the diagonal matrix whose elements are $\left[\exp \left(a_{1}\right), \ldots, \exp \left(a_{n}\right)\right]$. 
is a $(2 M+1) \times(2 M+1)$ diagonal matrix and

$$
(\mathbf{C})_{k k}=\int_{-\infty}^{+\infty} \exp (-\mathrm{j} 2 \pi k x) f_{\delta}(x) \mathrm{d} x
$$

is the characteristic function of the random variable $\delta$, $C_{\delta}(s)$, sampled in $s=-\mathrm{j} 2 \pi k$. In particular when $\delta$ is a zero mean Gaussian random variable with variance $\sigma_{\delta}^{2}$, we have: $\mathbf{C}=\exp \left(\sigma_{\delta}^{2} \mathbf{W}^{2} / 2\right)$ and $(\mathbf{C})_{k k}=\exp \left(-2 \pi^{2} k^{2} \sigma_{\delta}^{2}\right)$, $k=-M, \ldots, M$.

b) Computation of $\mathbb{E}\left[\mathbf{G}_{\mathbf{x}}^{\dagger} \mathbf{G}_{\mathbf{x}}\right]$ :

$$
\begin{aligned}
\mathbb{E}\left[\mathbf{G}_{\mathbf{x}}^{\dagger} \mathbf{G}_{\mathbf{x}}\right] & =\mathbb{E}\left[\sum_{\substack{n=0 \\
m=0}}^{\infty} \frac{1}{n ! m !} \boldsymbol{\Delta}^{n} \mathbf{G}_{\hat{\mathbf{x}}}^{\dagger} \mathbf{W}^{\dagger n} \mathbf{W}^{m} \mathbf{G}_{\hat{\mathbf{x}}} \boldsymbol{\Delta}^{m}\right] \\
& =\sum_{\substack{n=0 \\
m=0}}^{\infty} \frac{1}{n ! m !} \mathbb{E}\left[\boldsymbol{\Delta}^{n} \mathbf{Z} \boldsymbol{\Delta}^{m}\right]
\end{aligned}
$$

where $\mathbf{Z}=\mathbf{G}_{\hat{\mathbf{x}}}^{\dagger} \mathbf{W}^{\dagger n} \mathbf{W}^{m} \mathbf{G}_{\hat{\mathbf{x}}}$. Now,

$$
\begin{aligned}
\left(\mathbb{E}\left[\boldsymbol{\Delta}^{n} \mathbf{Z} \boldsymbol{\Delta}^{m}\right]\right)_{h k} & =\mathbb{E}\left[\delta_{h}^{n} \delta_{k}^{m}\right](\mathbf{Z})_{h k} \\
& = \begin{cases}\mu^{(n)} \mu^{(m)}(\mathbf{Z})_{h k} & \text { if } h \neq k \\
\mu^{(n+m)}(\mathbf{Z})_{h k} & \text { if } h=k\end{cases}
\end{aligned}
$$

therefore

$$
\begin{aligned}
\mathbb{E}\left[\boldsymbol{\Delta}^{n} \mathbf{Z} \boldsymbol{\Delta}^{m}\right] & =\mu^{(n)} \mu^{(m)}(\mathbf{Z}-\operatorname{diag}(\mathbf{Z}))+\mu^{(n+m)} \operatorname{diag}(\mathbf{Z}) \\
& =\mu^{(n)} \mu^{(m)} \mathbf{Z}+\left(\mu^{(n+m)}-\mu^{(n)} \mu^{(m)}\right) \operatorname{diag}(\mathbf{Z})
\end{aligned}
$$

and

$$
\begin{aligned}
\mathbb{E}\left[\mathbf{G}_{\mathbf{X}}^{\dagger} \mathbf{G}_{\mathbf{X}}\right]=\sum_{\substack{n=0 \\
m=0}} & \frac{\mu^{(n)} \mu^{(m)}}{n ! m !} \mathbf{Z} \\
& +\sum_{\substack{n=0 \\
m=0}}^{\infty} \frac{\mu^{(n+m)}-\mu^{(n)} \mu^{(m)}}{n ! m !} \operatorname{diag}(\mathbf{Z}) .
\end{aligned}
$$

The first term of (42) yields

$$
\begin{aligned}
\sum_{\substack{n=0 \\
m=0}}^{\infty} \frac{\mu^{(n)}}{n !} \frac{\mu^{(m)}}{m !} \mathbf{Z} & =\sum_{\substack{n=0 \\
m=0}}^{\infty} \frac{\mu^{(n)}}{n !} \frac{\mu^{(m)}}{m !} \mathbf{G}_{\hat{\mathbf{x}}}^{\dagger} \mathbf{W}^{\dagger n} \mathbf{W}^{m} \mathbf{G}_{\hat{\mathbf{x}}} \\
& =\mathbf{G}_{\hat{\mathbf{x}}}^{\dagger}\left[\sum_{n=0}^{\infty} \frac{\mu^{(n)}}{n !} \mathbf{W}^{n}\right]^{\dagger}\left[\sum_{m=0}^{\infty} \frac{\mu^{(m)}}{m !} \mathbf{W}^{m}\right] \mathbf{G}_{\hat{\mathbf{x}}} \\
& =\mathbf{G}_{\hat{\mathbf{x}}}^{\dagger} \mathbf{C}^{\dagger} \mathbf{C} \mathbf{G}_{\hat{\mathbf{x}}}
\end{aligned}
$$

The $(k, k)$ th element of $\mathbf{Z}$ is given by

$$
\begin{aligned}
(\mathbf{Z})_{k k} & =\sum_{h}\left(\mathbf{G}_{\hat{\mathbf{x}}}^{\dagger}\right)_{k h}\left(\mathbf{W}^{\dagger n} \mathbf{W}^{m}\right)_{h h}\left(\mathbf{G}_{\hat{\mathbf{x}}}\right)_{h k} \\
& =\sum_{h}\left|\left(\mathbf{G}_{\hat{\mathbf{x}}}\right)_{h k}\right|^{2}\left(\mathbf{W}^{\dagger n} \mathbf{W}^{m}\right)_{h h} \\
& =\frac{1}{2 M+1} \operatorname{Tr}\left\{\mathbf{W}^{\dagger n} \mathbf{W}^{m}\right\}
\end{aligned}
$$

which does not depend on $k$. Thus, $\operatorname{diag}(\mathbf{Z})=(1 /(2 M+$ 1)) $\operatorname{Tr}\left\{\mathbf{W}^{\dagger n} \mathbf{W}^{m}\right\} \mathbf{I}$, and

$$
\begin{aligned}
& \sum_{\substack{n=0 \\
m=0}}^{\infty} \frac{\mu^{(n)}}{n !} \frac{\mu^{(m)}}{m !} \operatorname{diag}(\mathbf{Z}) \\
& \quad=\frac{1}{2 M+1} \operatorname{Tr}\left\{\left[\sum_{n=0}^{\infty} \frac{\mu^{(n)}}{n !} \mathbf{W}^{n}\right]^{\dagger}\left[\sum_{m=0}^{\infty} \frac{\mu^{(m)}}{m !} \mathbf{W}^{m}\right]\right\} \mathbf{I} \\
& =\frac{1}{2 M+1} \operatorname{Tr}\left\{\mathbf{C}^{\dagger} \mathbf{C}\right\} \mathbf{I}
\end{aligned}
$$

Finally

$$
\begin{aligned}
& \sum_{\substack{n=0 \\
m=0}}^{\infty} \frac{\mu^{(n+m)}}{n ! m !} \operatorname{diag}(\mathbf{Z}) \\
& \quad=\frac{1}{2 M+1} \operatorname{Tr}\left\{\sum_{\substack{n=0 \\
m=0}}^{\infty} \frac{\mu^{(n+m)}}{n ! m !} \mathbf{W}^{\dagger n} \mathbf{W}^{m}\right\} \mathbf{I} \\
& =\frac{1}{2 M+1} \operatorname{Tr}\{\mathbf{Y}\} \mathbf{I}
\end{aligned}
$$

where

$$
\begin{aligned}
\mathbf{Y}= & \sum_{\substack{n=0 \\
m=0}}^{\infty} \frac{\mu^{(n+m)}}{n ! m !} \mathbf{W}^{\dagger n} \mathbf{W}^{m} \\
= & \sum_{\substack{n=0 \\
m=0}}^{\infty} \frac{1}{n ! m !} \int_{-\infty}^{+\infty} x^{n+m} f_{\delta}(x) \mathrm{d} x \mathbf{W}^{\dagger n} \mathbf{W}^{m} \\
= & \int_{-\infty}^{+\infty}\left[\sum_{n=0}^{\infty} \frac{x^{n} \mathbf{W}^{n}}{n !}\right]^{\dagger}\left[\sum_{m=0}^{\infty} \frac{x^{m} \mathbf{W}^{m}}{m !}\right] f_{\delta}(x) \mathrm{d} x . \\
= & \int_{-\infty}^{+\infty} \exp (x \mathbf{W})^{\dagger} \exp (x \mathbf{W}) f_{\delta}(x) \mathrm{d} x \\
= & \int_{-\infty}^{+\infty} \mathbf{I} f_{\delta}(x) \mathrm{d} x=\mathbf{I}
\end{aligned}
$$

Therefore

$$
\sum_{\substack{n=0 \\ m=0}}^{\infty} \frac{\mu^{(n+m)}}{n ! m !} \operatorname{diag}(\mathbf{Z})=\frac{1}{2 M+1} \operatorname{Tr}\{\mathbf{I}\} \mathbf{I}=\mathbf{I}
$$

Concluding, if $\mathbf{C}$ is real,

$$
\mathbb{E}\left[\mathbf{G}_{\mathbf{x}}^{\dagger} \mathbf{G}_{\mathbf{x}}\right]=\mathbf{G}_{\hat{\mathbf{x}}}^{\dagger} \mathbf{C}^{2} \mathbf{G}_{\hat{\mathbf{x}}}+\left(1-\frac{\operatorname{Tr}\left\{\mathbf{C}^{2}\right\}}{2 M+1}\right) \mathbf{I} .
$$

\section{ACKNOWLEDGMENT}

The authors would like to thank the reviewers who, by their careful reading and constructive comments, have greatly enhanced the value of this manuscript. 


\section{REFERENCES}

[1] I. F. Akyildiz, W. Su, Y. Sankarasubramaniam, and E. Cayirci, "Wireless sensor networks: A survey," Comp. Networks, vol. 38, no. 4, pp. 393-422, 2002.

[2] D. Moore, J. Leonard, D. Rus, and S. Teller, "Robust distributed network localization with noisy range measurements," in Proc. 2nd ACM Conf. Embedded Netw. Sens. Syst. (SenSys '04), Baltimore, MD, Nov. 2004, pp. 50-61.

[3] D. Ganesan, S. Ratnasamy, H. Wang, and D. Estrin, "Coping with irregular spatio-temporal sampling in sensor networks," in Proc. 2nd Workshop on Hot Topics in Netw. (HotNets-II), Cambridge, MA, Nov. 2003.

[4] H. G. Feichtinger, K. Gröchenig, and T. Strohmer, "Efficient numerical methods in non-uniform sampling theory," Numerische Mathematik, vol. 69, pp. 423-440, 1995.

[5] K. Gröchenig, "Irregular sampling, Toeplitz matrices, and the approximation of entire functions of exponential type," Math. Computat., vol. 68, no. 226, pp. 749-765, Apr. 1999.

[6] F. A. Marvasti, Nonuniform Sampling: Theory and Practice. New York: Kluwer Academic: Plenum, 2001.

[7] The Autonomous Ocean Sampling Network (AOSN) Project [Online]. Available: http://www.mbari.org/aosn/

[8] E. Cayirci, H. Tezcan, Y. Dogan, and V. Coskun, "Wireless sensor networks for underwater surveillance systems," Ad Hoc Netw., vol. 4, no. 4, pp. 431-446, Jul. 2006.

[9] S. J. Majumdar et al., "A comparison of adaptive observing guidance for Atlantic tropical cyclones," in Proc. 27th Conf. Hurricanes and Trop. Meteorol., Apr. 2006.

[10] M. J. Wainwright, Sharp Thresholds for High-Dimensional and Noisy Recovery of Sparsity [Online]. Available: http://www.arxiv.org/abs/ math/0605740

[11] S. Cambanis and E. Masry, "Sampling designs for the detection of signals in noise," IEEE Trans. Inf. Theory, vol. 29, pp. 83-104, Jan. 1983.

[12] J. M. Hammersley and D. C. Handscomb, The Monte Carlo Method. London, U.K.: Methuen, 1964.

[13] M. C. Vuran, Ö. B. Akan, and I. F. Akyildiz, "Spatio-temporal correlation: Theory and applications for wireless sensor networks," Сomp. Netw., vol. 45, no. 3, pp. 245-259, Jun. 2004.

[14] S. C. Ergen and P. Varaiya, "Effects of A-D conversion nonidealities on distributed sampling in dense sensor networks," in IPSN, Nashville, TN, Apr. 2006.

[15] B. Liu and T. P. Stanley, "Error bounds for jittered sampling," IEEE Trans. Autom. Control, vol. 10, no. 4, pp. 449-454, Oct. 1965.

[16] M. Perillo, Z. Ignjatovic, and W. Heinzelman, "An energy conservation method for wireless sensor networks employing a blue noise spatial sampling technique," in IPSN, Apr. 2004.

[17] P. Ishwar, A. Kumar, and K. Ramchandran, "Distributed sampling for dense sensor networks: A bit-conservation principle," in IPSN, Apr. 2003.

[18] Z. Yang, M. Dong, L. Tong, and B. M. Sadler, "MAC protocols for optimal information retrieval pattern in sensor networks with mobile access," EURASIP J. Wireless Commun. Netw., vol. 5, no. 4, pp. 493-504, Sep. 2005.

[19] M. Dong, L. Tong, and B. M. Sadler, "Impact of data retrieval pattern on homogeneous signal field reconstruction in dense sensor networks," IEEE Trans. Signal Process., vol. 54, no. 11, pp. 4352-4364, Nov. 2006.

[20] R. Cristescu and M. Vetterli, "On the optimal density for real-time data gathering of spatio-temporal processes in sensor networks," in IPSN, Los Angeles, CA, Apr. 2005.

[21] Y. Sung, L. Tong, and H. V. Poor, "Sensor activation and scheduling for field detection in large sensor arrays," in IPSN, Los Angeles, CA, Apr. 2005.

[22] Y. Rachlin, R. Negi, and P. Khosla, "Sensing capacity for discrete sensor network applications," in IPSN, Los Angeles, CA, Apr. 2005.

[23] P. Marziliano and M. Vetterli, "Reconstruction of irregularly sampled discrete-time bandlimited signals with unknown sampling locations," IEEE Trans. Signal Process., vol. 48, no. 12, pp. 3462-3471, Dec. 2000.

[24] P. Stoica and R. Moses, Introduction to Spectral Analysis. Upper Saddle River, NJ: Prentice-Hall, 2000.

[25] H. Rauhut, Random Sampling of Sparse Trigonometric Polynomials [Online]. Available: http://www.homepage.univie.ac.at/holger.rauhut/ RandomSampling.pdf

[26] H. G. Feichtinger and K. Gröchenig, "Error analysis in regular and irregular sampling theory," Applicable Anal., vol. 50, pp. 167-189, 1993.
[27] H. Rauhut, Stability Results for Random Sampling of Sparse Trigonometric Polynomials [Online]. Available: http://www.homepage.univie.ac.at/holger.rauhut/StabilityRS.pdf

[28] E. Candes and T. Tao, The Dantzig Selector: Statistical Estimation When $\mathrm{p}$ is Much Larger Than n [Online]. Available: http://www.acm. caltech.edu/emmanuel/papers/DantzigSelector.pdf

[29] A. Aldroubi and K. Gröchenig, "Nonuniform sampling and reconstruction in shift-invariant spaces," SIAM Rev., vol. 43, pp. 585-620, Dec. 2001.

[30] A. Nordio, C.-F. Chiasserini, and E. Viterbo, "Signal reconstruction in multidimensional sensor fields," in IZS 2008, Zurich, Switzerland, Mar. 12-14, 2008.

[31] S. Verdù, Multiuser Detection. Cambridge, U.K.: Cambridge Univ. Press, 1998.

[32] A. Tulino and S. Verdú, "Random matrix theory and wireless communications," Found. Trends in Commun. Inf. Theory, vol. 1, no. 1, 2004.

[33] E. Biglieri, G. Taricco, and A. Tulino, "Performance of space-Time codes for a large number of antennas," IEEE Trans. Inf. Theory, vol. 48, no. 7, pp. 1794-1803, July 2002.

[34] M. Brookes, The Matrix Reference Manual. London, U.K.: Imperial College, 1998.

[35] R. Bellman, Introduction to Matrix Analysis, 2nd ed. New York: McGraw-Hill, 1970.

[36] W. H. Press, S. A. Teukolsky, W. T. Vetterling, and B. P. Flannery, Numerical Recipes, 2nd ed. Cambridge, U.K.: Cambridge Univ. Press, 1997.

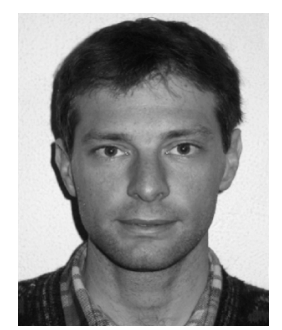

Alessandro Nordio (S'00-M'03) received the Laurea degree in telecommunications engineering from the Politecnico di Torino, Italy, in 1998, and the $\mathrm{Ph} . \mathrm{D}$. degree from École Politechnique Fédérale de Lausanne, Switzerland, in 2002.

He then joined the Department of Electrical Engineering, Politecnico di Torino, where he is working as a Postdoctoral Researcher. His research interests are in the field of signal processing, multiuser detection, space-time coding, sensor networks, and theory of random matrices.

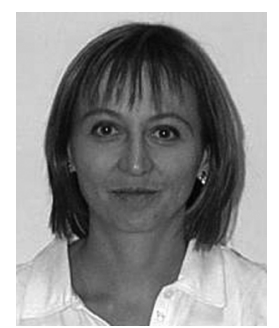

Carla-Fabiana Chiasserini (M'98) received the Laurea degree from the University of Florence, Florence, Italy, in 1996 and the Ph.D. degree from Politecnico di Torino, Italy, in 2000.

From 1998 to 2003, she has worked as a visiting researcher at the University of California at San Diego. She is currently an Associate Professor at Politecnico di Torino.

Dr. Chiasserini is a member of the editorial board of the Ad Hoc Networks Journal (New York: Elsevier), and has served as an Associate Editor of the IEEE COMMUNICATIONS LETTERS since 2004.

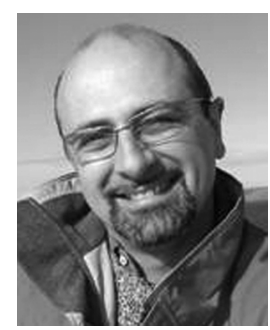

Emanuele Viterbo (M'95-SM'04) received the Laurea degree in 1989 and the Ph.D. degree in 1995 , in electrical engineering, from the Politecnico di Torino, Torino, Italy.

From 1990 to 1992, he was with the European Patent Office, The Hague, The Netherlands, as a patent examine. He became an Associate Professor with the Dipartimento di Elettronica, Politecnico di Torino, in 2005 and since November 2006, has been a Full Professor at the Università della Calabria, Italy.

Dr. Viterbo was awarded a NATO Advanced Fellowship in 1997 from the Italian National Research Council. He is an Associate Editor of the IEEE TRANSACTIONS ON INFORMATION THEORY, European Transactions on Telecommunications, and the Journal of Communications and Networks. 